\title{
Nonlinear dynamics and control of a set of robots for hunting and coverage missions
}

\author{
H. Sayyaadi · M. T. Sabet
}

Received: 25 July 2013 / Revised: 7 March 2014 / Accepted: 12 March 2014 / Published online: 5 April 2014

(C) Springer-Verlag Berlin Heidelberg 2014

\begin{abstract}
The aim of this article is control of a set of 3wheel robots with non-holonomic dynamics for hunting and covering around moving target. Robots have mass and inertia, and the wheel mass have been considered in the dynamic model of robots. The output of the controller is wheel's torque and steering torque of the front wheel. The saturation and filtering effects of the actuators are also considered here. The robots in the group are controlled in such a way that each robot responds with an appropriate reaction, based on the control algorithm and the information passed down from other robots and the target (decentralized control). Moving target dynamics has been considered in a way that it is escaping from invader, and the target has holonomics dynamic, and it is assumed that the moving target hasn't the wheel. To derive the equation of the motion, Kane dynamics procedure has been used. Robots are equipped with sensors for distance assessment, vision angle assessment and also signal receiver antenna. To estimate relative position and variables of another robot situation and target, Extended Kalman filter and Extended Kalman smoother (Extended Rauch-Tung-Strible smoother) had been used. To do a group maneuver using inertia analysis and optimizing the norm of error between desired and actual acceleration, controller was designed. The operation was implemented for three planes: ramp plane, spherical plane and cylindrical plane. The results include the hunting and the coverage of the target by four invader robot and relative distance diagrams between robots and the target, their velocity and corresponding to robots at three planes and also a comparison between real and estimated variables and moreover,comparison between estimations by Kalman filter and Kalman smoother. Based on the results,
\end{abstract}

H. Sayyaadi · M. T. Sabet $(\bowtie)$

School of Mechanical Engineering, Sharif University of Technology, Tehran, Iran

e-mail: mohammadtaghi.sabet@gmail.com it can be seen that due to inertia effects in robots and nonholonomics feature of robots, designed controller and estimator are suitable for implementing to the operation to achieve appropriate results.

Keywords Multiagent system - Non-Holonomic dynamic . Hunting and coverage - Kalman filter - Kalman Smoother . Uneven plans

\section{Introduction}

In human environments, we have a network of individuals who are participating with each other for different reasons. So, for making a society form different agents, it is required to make a relation between them and these agents must be coordinated with each other. This coordination can have different parts for commission or competition. These societies are called multi agent systems.

Multi agent systems are made by gathering of coordinated agents and the relations between them. In these systems, everyone knows its duty and knows when to communicate with the other one. The nature of these systems is based on this rule that a group of agents with common capabilities are replaced by an agent by supernatural capability. The study of these systems because of their velocity, reliability, maintenance, reflex ability, changeability and extendibility to divide the woks between agents is very important [1].

The application of these systems is very popular among human life such as moving robot systems and sensor's networks. Recently, improvement in wireless networks, production of high-velocity processors and production of miniature moving robots with different sensors, has extended the application of these systems $[2,3]$. 
Among different robots, wheeler robots are very significant. One of the wheeler robots is a tricycle robot that because of simple making, special geometrical shape, dynamic balance, capability for installation of instruments and carrying of load, low maintenance cost, suitable suspension system and implementing group maneuvers with high velocity is very important. In this paper, this robot is used to hunt and follow missions that it can be used in military and also scientific applications. Meanwhile, it can be used for making a security follow for a leader. One of the works done in this paper is surveying the movement of this type of robots in non-flat surfaces that this work shows the implicational actuality of these systems.

In addition to the non-Holonomic kinematic, of this kind of robots are considered with their mass and moment of inertia, and we have surveyed robots dynamically. In this paper, the equation of motion of the robots is derived by Kane Method that it is important in control law designing. In control law, any robot is controlled by relative distance and velocity with other robots and the target. The control law is decentralized and scalable. It is not limited to the number of robots.

It is supposed that each robot use of the estimator. Every robot estimates the target and other robots position and velocity. Each robot by using of the output of its sensors and using Kalman filter, estimates the required state variables and also for optimizing the estimate uses Kalman smoother and the mission is implemented based on these estimates. To control the robots are implemented special control law, relative to each mission.

For compare by ago works, in [4] design and implements a non-holonomic wheeled mobile robot that possesses dynamic object-tracking capability by using real-time image processing. Two motion control laws are proposed using Lyapunov's direct method and computed-torque method. The problem of navigation and guidance of a Dubins-like wheeled robot towards a steady or maneuvering target with rangeonly information. We propose a range-based sliding mode controller through which the robot follows a target with a constant speed while preserving a predefined range margin from the target [5]. Meanwhile by using of fuzzy control for passing of the robot next to the obstacle and route creating [6], and passing from moving and stable obstacle and also passing from the small corridor that in both references, fuzzy control was used and robots controlled kinematically [7,8].

The work done about the group mission of wheeler robots are discussed at follow. A distributed smooth timevarying feedback control law for coordinating motions of multiple non-holonomic mobile robots of the Hilare-type to capture/enclose a target by making troop formations. This motion coordination is a cooperative behavior for security against invaders in surveillance areas. Each robot in this control law has its own coordinate system, and it senses a tar- get/invader, other robots and obstacles, to achieve this cooperative behavior without making any collision. Each robot especially has a two-dimensional control input referred to as a "formation vector" and the formation is controllable by the vectors [9].

The purpose is control and estimate the state variables of three car-like robots for implementing the group mission with the special arrangement group mission has been without leader and Kalman estimator has been used for estimating relative distances with other robots and the obstacles. The control of the system has been surveyed kinematically [10].

The reference [11] is a doctorate thesis, the target is controlling a number of car-like robots. In this survey, it is supposed that front wheels are parallel (In actuality it is not the same), and robot with two wheels was analyzed kinematically. Cost function is the difference in actual velocity and desired velocity that it is optimized by a special control signal. There is another view in which Euclidean normcost function - optimizes the difference in actual and desired place that its control is based on (MPC) which is liberalized for the model and also its non- linearization form (NMPC). It is supposed; each robot has one leader, and this leader continues toward the main leader.

Therefore, the control algorithm for each robot was defined such that they follow the leader at a certain relative distance and angle [12]. In this survey, follow robot moves in an optimized direction toward the leader robot. It is supposed that state variables are not obvious and estimator has been used. The WIFI data includes the reception signal strength (RSS) and the angle of the maximal RSS with respect to robot's orientation, which are used for finding the relative place of the robots $[13,14]$.

To control the system a kinematic system has been used and also mass and moment of inertia and inputting torque of the system are ignored. It is supposed that the main leader has a constant velocity, thus it is not the same as control algorithms of accelerating systems. Meanwhile, the control algorithm was based on an optimization procedure in which the cost function using numerical methods uses a lot of CPU time of the system. Ref. [25] is motion control of a wheeled mobile robot using digital acceleration control method that in this, paper is used from a proportional-integrator controller.

The implementation of this algorithm requires to predict of future, numerical optimization and doing many calculations. [15] In general, the control of the systems is divided to two categories: kinematic control and dynamic control. Among them, dynamic control has special importance, and it is due to considering of force or torque as the controller that in this paper, it is supposed that state variables are not obvious and estimator has been used. To do group control, a procedure has been used that is not central and do not have the leader who increases the types of motions and also the algorithm is in a manner that we can add a number of robots 
and obstacles to it. Meanwhile, hunt mission on non-planer surfaces in special has been studied. Control algorithm is simple, and the surface of calculations is low. In the following, we analyze the kinematic and dynamic of the robot in three surfaces: inclined surface, spherical surface and cylindrical surface, then we study the design of a robot controller, describing dynamic of the target, design of group control for implementing of the mission, designing state estimator for robots and target, introducing diagrams and results, conclusion, references, and at last we provide an index.

\section{Dynamic and kinematic analyzes of robot}

As, it can be seen in Fig. 1, the applied robot has the tricycle. The wheels have also mass and moment of inertia, and only we have ignored the mass of the front wheel for suspension. The steering's torque and front wheel's torque are considered as controllers.

In Fig. 1, generalized coordinates are introduced for robot that is used for each three surfaces. The only difference in these three surfaces is related to definition of generalized coordinates of the center of robots that they are at three different surfaces, and they are defined depending on the kind of surfaces such as Cartesian, spherical and cylindrical.

In this figure, is the body angle of the robot, is the steering angle, is the velocity of the front wheel, is the distance between the center of the front wheel and center of the rear axis, is a symbol for the geometrical center of the robot body, is the distance between geometrical center and the center of the rear axis, is the distance between the center of the body axis and each of the rear wheels, and are angels for circulation of left and right wheels in the rear, is the radius of the front

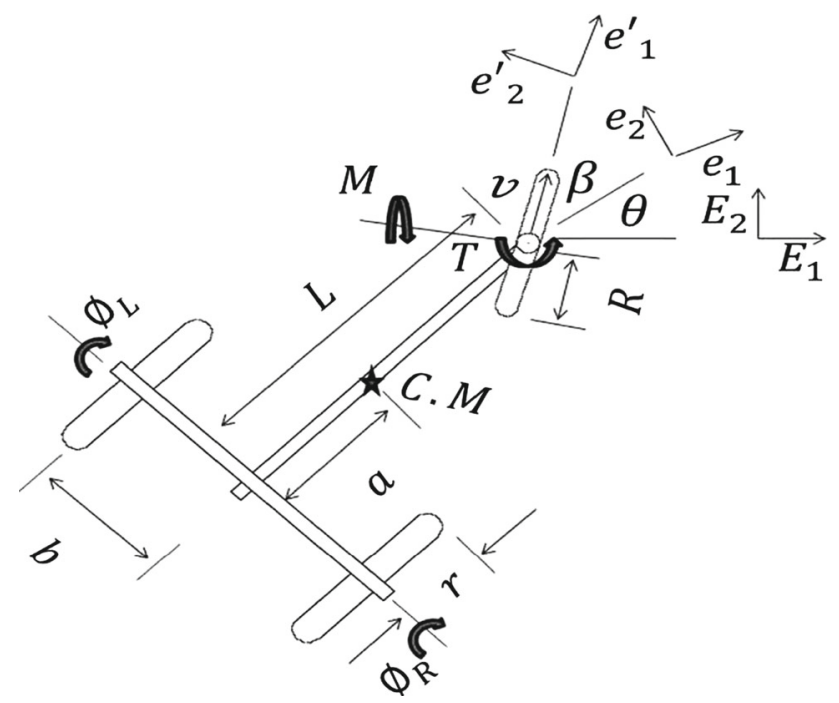

Fig. 1 Schematic diagram of a tricycle robot with Generalized coordinates wheel, is the radius of the rear wheels, and are the torque of the front wheel and the steering torque, respectively.

The body of the robot has mass and moment of inertia equal to and, respectively. The rear wheels have a mass equal to, and moment of inertia equal to in different directions that symbol shows the direction of the coordinates. To analyze dynamic and kinematic motion, Kane dynamic procedure has been used $[16,17]$.

\subsection{Equation of motion in ramp surface}

We consider the inclined surface with an inclination equal $\alpha$. In Fig. 2, the generalized coordinates and external forces of the robot are shown.

First of all, we introduce the transformation of coordinates systems that they are used in Eqs. (1) and (2) are important alterations in calculating of equations of motion. In following equations, $e$ is a symbol for unit vector of each coordinates systems and also in this paper, Sin and Cos are referred to as $S$ and $C$.

$$
\begin{aligned}
& {\left[\begin{array}{l}
e_{x^{\prime}} \\
e_{y^{\prime}} \\
e_{z^{\prime}}
\end{array}\right]=\left[\begin{array}{ccc}
C \beta & S \beta & 0 \\
-S \beta & C \beta & 0 \\
0 & 0 & 1
\end{array}\right]\left[\begin{array}{l}
e_{x} \\
e_{y} \\
e_{z}
\end{array}\right]} \\
& {\left[\begin{array}{l}
e_{X^{\prime}} \\
e_{Y^{\prime}} \\
e_{Z^{\prime}}
\end{array}\right]=\left[\begin{array}{ccc}
C \theta & -S \theta & 0 \\
S \theta & C \theta & 0 \\
0 & 0 & 1
\end{array}\right]\left[\begin{array}{l}
e_{x} \\
e_{y} \\
e_{z}
\end{array}\right]}
\end{aligned}
$$

Because, we use Kane dynamic procedure for calculating dynamic of the systems, first of all, we must select quasi velocities for solving. We must pay more attention for selecting them to use them in dynamic calculates and slow in control of the system, because, these quasi velocities enter in the final equations, and they must be controlled. Thus, we select the velocity of the front wheel as well as angle velocity of the steering as quasi velocities. In the section of robot's con-

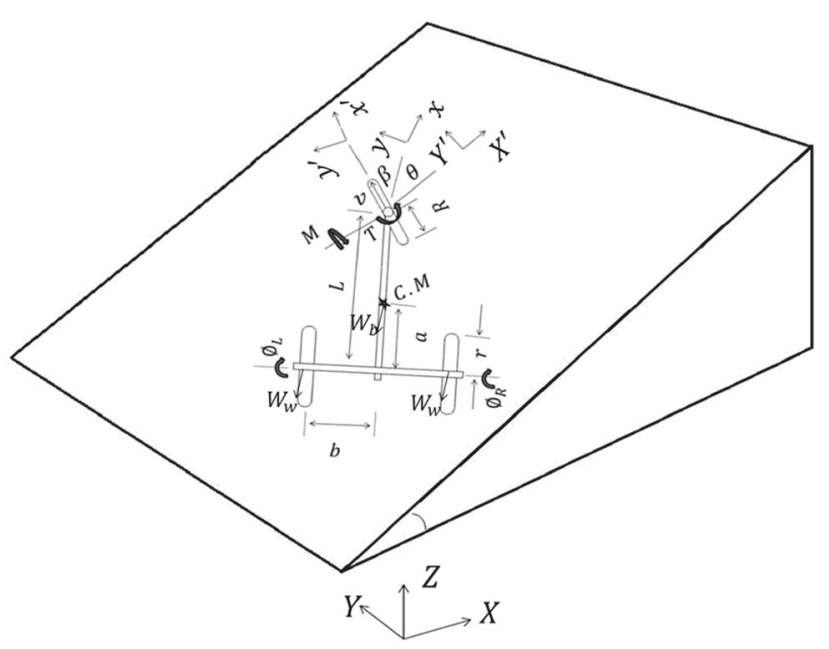

Fig. 2 Generalized coordinates in ramp surface 
trol, we can see its importance. Thus $u_{1}=v$ and $u_{2}=\dot{\beta}$ are selected as two quasi velocity at three surfaces. We calculate the constrain equations based on non-slipping conditions in wheels. It must be noticed because we have used Kane dynamic procedure; it was required to calculate constrain equations based on quasi velocities. To do this, it was required to find the velocity of the mass center based on front wheel's velocity. In follow, we introduce calculating the constrain equations.

$$
\begin{aligned}
v_{A} & =u_{1} e_{x^{\prime}} \\
v_{R} & =v_{A}-L \dot{\theta} e_{y}+b \dot{\theta} e_{x}=r \dot{\phi}_{R} e_{x} \\
v_{L} & =v_{A}-L \dot{\theta} e_{y}-b \dot{\theta} e_{x}=r \dot{\phi}_{L} e_{x} \\
v_{b} & =v_{A}-(L-a) \dot{\theta} e_{y}=\dot{X}^{\prime} e_{X^{\prime}}+\dot{Y}^{\prime} e_{Y^{\prime}}
\end{aligned}
$$

In these equations, $v_{A}$ is the velocity of the front wheel, $v_{R}$ and $v_{L}$ are rear wheels' velocities for right and left wheels, respectively and $v_{b}$ is the velocity of the robot body center. To find the acceleration of each component, we can differentiate the velocity.

Now, the equations will become as follows. As, we can see these equations are non-holonamic and we can't get integral from them. Constrain equations like $a_{i}^{T}(q) \dot{q}=0$ are called Pfaffian. In Eq. (7) some state variables are introduced.

$a_{1}=C \beta C \theta-\frac{a}{L} S \beta S \theta$

$a_{2}=C \beta S \theta+\frac{a}{L} S \beta C \theta, \quad a_{3}=\frac{S \beta}{L}$

$a_{4}=\frac{C \beta}{r}+\frac{b}{r L} S \beta, \quad a_{5}=\frac{C \beta}{r}-\frac{b}{r L} S \beta$

Constrain equations:

$$
\begin{aligned}
& \dot{X}^{\prime}=a_{1} u_{1} \\
& \dot{Y}^{\prime}=a_{2} u_{1} \\
& \dot{\theta}=a_{3} u_{1} \\
& \dot{\phi}_{R}=a_{4} u_{1} \\
& \dot{\phi}_{L}=a_{5} u_{1}
\end{aligned}
$$

To solve based on Kane dynamic procedure, we need to angular velocity, angular momentum and its differentiation. Angular Velocity is introduced in the following equations, and the angular momentum can be calculated by multiplying of angular velocity of each component in the moment of inertia. $(H=I \omega)$

$$
\begin{aligned}
\omega_{R} & =\dot{\phi}_{R} e_{y}+\dot{\theta} e_{z} \\
\omega_{L} & =\dot{\phi}_{L} e_{y}+\dot{\theta} e_{z} \\
\omega_{b} & =\dot{\theta} e_{z}
\end{aligned}
$$

Now, by using of Kane, dynamic equation introduced in Eq. (16), we will find robot's equation of motion. In this equation, $V_{G_{i}}^{k}$ is velocity vector related to each of the quasi velocities in systems. $a_{G_{i}}$ Is the acceleration of the mass center of each component, $\omega_{G_{i}}^{k}$ is the angular velocity vector of each of the components, $F_{G}$ and $M_{G}$ are force and entered torque over the mass center of each of the components and $F_{B}$ is the entered force over each of the components out of the mass center.

$$
\begin{aligned}
\sum_{i=1}^{N} V_{G_{i}}^{k} \cdot m a_{G_{i}}+\omega_{G_{i}}^{k} \cdot \dot{H}_{G}= & \sum_{i=1}^{N} V_{G_{i}}^{k} \cdot F_{G_{i}} \\
& +\omega_{G_{i}}^{k} \cdot M_{G}+V_{B_{i}}^{k} \cdot F_{B}
\end{aligned}
$$

Dynamic equations of motion change as follows by using of Kane dynamic equations. The coefficients of $a_{i}$ state variables in this equation are parameters related to the steering angle and robot body that they are introduced in the Appendix 1.

$\dot{u}_{1}=\frac{M}{R a_{9}}+\frac{a_{12}}{a_{9}}-\frac{a_{10}}{a_{9}} u_{1} u_{2}-\frac{a_{11}}{a_{9}} u_{1}^{2}$

$u_{2}=\frac{T}{C_{b}}$

To Find $X^{\prime}$ and $Y^{\prime}$ we can take differential from them because Eqs. (8) and (9) are implemented continuously that they become as following equations. In Eqs. (19) and (20), $a_{i}$ state variables are available that they are in Appendix 1.

$\ddot{X}^{\prime}=\dot{u}_{1} a_{1}+u_{1} a_{13}$

$\ddot{Y}^{\prime}=\dot{u}_{1} a_{2}+u_{1} a_{14}$

By selecting state variables as follows, we can write the equations based on the state space form.

$x_{1}=u_{1}, \quad x_{2}=\beta, \quad x_{3}=\theta, \quad x_{4}=X^{\prime}, \quad x_{5}=Y^{\prime}$

Equations of state space for robot's motion:

$$
\left[\begin{array}{l}
\dot{x}_{1} \\
\dot{x}_{2} \\
\dot{x}_{3} \\
\dot{x}_{4} \\
\dot{x}_{5}
\end{array}\right]=\left[\begin{array}{l}
-\frac{a_{11}}{a_{9}} x_{1}^{2}+\frac{a_{12}}{a_{9}} \\
0 \\
a_{3} x_{1} \\
a_{1} x_{1} \\
a_{2} x_{1}
\end{array}\right]+\left[\begin{array}{cc}
\frac{1}{R a_{9}}-\frac{a_{10}}{a_{9} C_{b}} x_{1} \\
0 & 1 / C_{b} \\
0 & 0 \\
0 & 0 \\
0 & 0
\end{array}\right]\left[\begin{array}{l}
M \\
T
\end{array}\right]
$$

We can write it in abbreviation as follow:

$$
\begin{aligned}
& \dot{X}=f(X, \tau) \\
& X=\left[x_{1} \cdots x_{5}\right]^{T}, \quad \tau=[M T]^{T}
\end{aligned}
$$

\subsection{Equations of motion in cylindrical surface}

In the cylindrical surface for finding the equations of motion, we can do it as ramp surface and the only difference in this surface is that cylindrical coordinate is used in place of cartesian. Generalized coordinates of the robot in this surface are shown in Fig. 3. It should be noticed that for finding the equations of motion, it was supposed that the radius of the surface has been big enough until in one point of the surface, all the components of the robot stay in one height. 


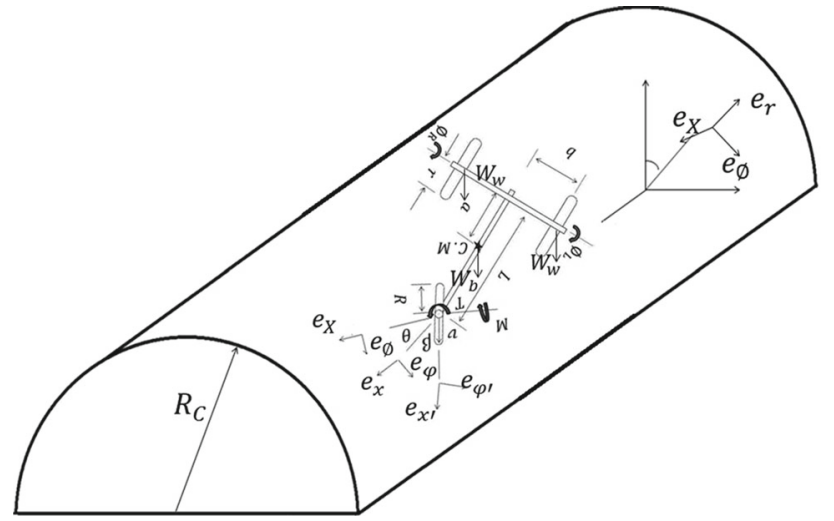

Fig. 3 Generalized coordinates in cylindrical surface

Matrixes for the coordinate change are defined as following equations in cylindrical coordinate.

$\left[\begin{array}{l}e_{\varphi} \\ e_{x}\end{array}\right]=\left[\begin{array}{cc}C \theta & -S \theta \\ S \theta & C \theta\end{array}\right]\left[\begin{array}{l}e_{\phi} \\ e_{X}\end{array}\right]$
$\left[\begin{array}{l}e_{\varphi^{\prime}} \\ e_{x^{\prime}}\end{array}\right]=\left[\begin{array}{cc}C \beta & -S \beta \\ S \beta & C \beta\end{array}\right]\left[\begin{array}{l}e_{\varphi} \\ e_{x}\end{array}\right]$
$\left[\begin{array}{l}e_{X} \\ e_{Y} \\ e_{Z}\end{array}\right]=\left[R_{\phi, e_{X}}\right]\left[R_{-\theta, e_{r}}\right]\left[\begin{array}{l}e_{x} \\ e_{y} \\ e_{z}\end{array}\right]$
$\left[R_{\phi, e_{X}}\right]\left[R_{-\theta, e_{r}}\right]=\left[\begin{array}{ccc}C \theta & S \theta & 0 \\ -S \theta C \phi & C \phi C \theta & -S \phi \\ -S \phi S \theta & S \phi C \theta & C \phi\end{array}\right]$

Like ramp surface if there is no slipping condition, we can find the kinematic constrains. It should be noticed that the velocity of each of the components is in the Appendix 2.

Motion constrains:

$\dot{X}=a_{1} u_{1}$

$\dot{\phi}=\frac{a_{2} u_{1}}{R_{c}+r}$

Three other motion constrains are calculated like Eqs. (10), (11) and (12).

Now, for finding the equations of motion based on Kane dynamic procedure as it was discussed about the ramp surface equations; it is required to find the angular velocity that it is in Appendix 2 until by using it; we can calculate the angular momentum motion and also its differential.

By using of Kane dynamic equation that it is shown in Eq. (16), equations of motion can be calculated like Eqs. (30) and (31). It should be noticed that $a_{i}$ state variables are shown in Appendix 1.

$\dot{u}_{1}=\frac{M}{R a_{17}}+\frac{a_{20}}{a_{17}}-\frac{a_{18}}{a_{17}} u_{1} u_{2}-\frac{a_{19}}{a_{17}} u_{1}^{2}$

$u_{2}=\frac{T}{C_{b}}$ $\ddot{\phi}$ and $\ddot{X}$ can be calculated by differential of Eqs. (28) and (29) because these equations are implemented continuously.

$\ddot{X}=\dot{u}_{1} a_{1}+u_{1} a_{13}$

$\ddot{\phi}=\frac{\dot{u}_{1} a_{2}}{R_{C}+r}+\frac{u_{1} a_{14}}{R_{C}+r}$

Now we can write the equation of motion to the form of following state space. State variables are defined like Eq. (34).

$$
\begin{aligned}
& x_{1}=u_{1}, \quad x_{2}=\beta, \quad x_{3}=\theta, \quad x_{4}=X, \quad x_{5}=\phi \\
& {\left[\begin{array}{l}
\dot{x}_{1} \\
\dot{x}_{2} \\
\dot{x}_{3} \\
\dot{x}_{4} \\
\dot{x}_{5}
\end{array}\right]=\left[\begin{array}{l}
-\frac{a_{19}}{a_{17}} x_{1}^{2}+\frac{a_{20}}{a_{17}} \\
0 \\
a_{3} x_{1} \\
a_{1} x_{1} \\
\frac{a_{2} x_{1}}{R_{C}+r}
\end{array}\right]+\left[\begin{array}{cc}
\frac{1}{R a_{17}}-\frac{a_{18}}{a_{17} C_{b}} x_{1} \\
0 & 1 / C_{b} \\
0 & 0 \\
0 & 0 \\
0 & 0
\end{array}\right]\left[\begin{array}{l}
M \\
T
\end{array}\right]}
\end{aligned}
$$

this state space equation can be shown like total form of Eq. (23).

\subsection{Equations of motion in spherical surface}

To calculate equations of motion, we use spherical coordinates system. It should be noticed that for finding the equation of motion, it is supposed that, the surface radius is big enough that in one point of the surface, all the robot components stay in one height. Figure 4 shows the generalized coordinates of the robot.

Matrixes for transformation of coordinates can be defined as following equations based on spherical coordinate.

$$
\begin{aligned}
& {\left[\begin{array}{l}
e_{\delta} \\
e_{\gamma} \\
e_{r}
\end{array}\right]=\left[\begin{array}{lll}
C \theta & -S \theta & 0 \\
S \theta & C \theta & 0 \\
0 & 0 & 1
\end{array}\right]\left[\begin{array}{l}
e_{\delta^{\prime}} \\
e_{\gamma^{\prime}} \\
e_{\gamma^{\prime}}
\end{array}\right]} \\
& {\left[\begin{array}{l}
e_{\delta^{\prime \prime}} \\
e_{\gamma^{\prime \prime}} \\
e_{r^{\prime \prime}}
\end{array}\right]=\left[\begin{array}{ccc}
C \beta & S \beta & 0 \\
-S \beta & C \beta & 0 \\
0 & 0 & 1
\end{array}\right]\left[\begin{array}{l}
e_{\delta^{\prime}} \\
e_{\gamma^{\prime}} \\
e_{r^{\prime}}
\end{array}\right]}
\end{aligned}
$$

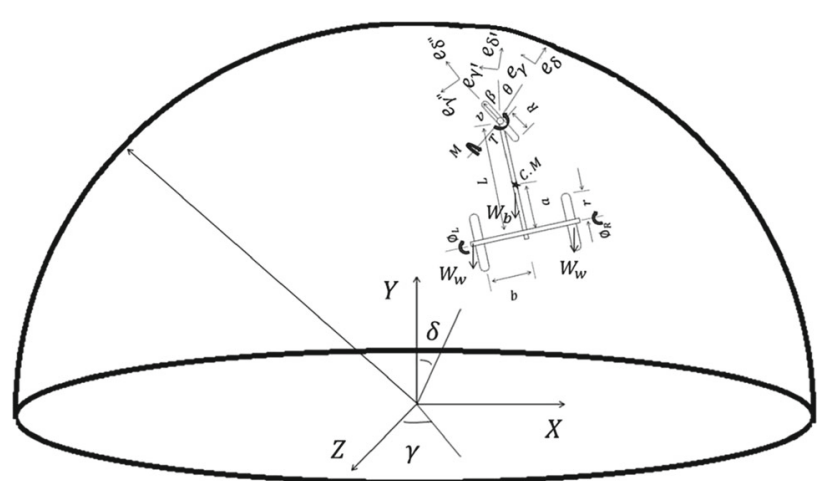

Fig. 4 Generalized coordinates in spherical surface 


$$
\begin{aligned}
& {\left[\begin{array}{l}
e_{X} \\
e_{Y} \\
e_{Z}
\end{array}\right]=\left[R_{-\gamma, Y}\right]\left[R_{-\delta, X}\right]\left[R_{-\theta, Y}\right]\left[\begin{array}{l}
e_{\gamma^{\prime}} \\
e_{r^{\prime}} \\
e_{\delta^{\prime}}
\end{array}\right]} \\
& {\left[R_{-\gamma, Y}\right]\left[R_{-\delta, X}\right]\left[R_{-\theta, Y}\right]} \\
& =\left[\begin{array}{lll}
C \gamma C \theta-S \theta C \delta S \gamma & -S \gamma S \delta & C \gamma S \theta+C \theta C \delta S \gamma \\
-S \theta S \delta & C \delta & S \delta C \theta \\
-S \gamma C \theta-S \theta C \delta C \gamma & -S \delta C \gamma & -S \gamma S \theta+C \theta C \delta C \gamma
\end{array}\right]
\end{aligned}
$$

By using of non-slipping condition for constrain equations, we can have following equations. The velocity of each component is shown in Appendix 2.

$\dot{\delta}=-\frac{a_{1} u_{1}}{R_{s}+r}$

$\dot{\gamma}=\frac{a_{2} u_{1}}{\left(R_{S}+r\right) S \delta}$

$\dot{\theta}=\left(a_{3}-\frac{a_{2} C \delta}{\left(R_{S}+r\right) S \delta}\right) u_{1}$

Two other constrain equations would be like Eqs. (11) and (12). Angular velocity of each component is shown in Appendix 2 that using it we can calculate angular momentum.

By using Kane dynamic equation that is shown in Eq. (16), equations of motion can be calculated like Eqs. (42) and (43). It should be noticed that $a_{i}$ state variables in these equations are shown in Appendix 1.

$\dot{u}_{1}=\frac{M}{R a_{21}}+\frac{a_{24}}{a_{21}}-\frac{a_{22}}{a_{21}} u_{1} u_{2}-\frac{a_{23}}{a_{21}} u_{1}^{2}$

$u_{2}=\frac{T}{C_{b}}$

$\ddot{\delta}$ and $\ddot{\gamma}$ can be calculated by differential of Eqs. (39) and (40) because these equations are implemented continuously.

$\ddot{\delta}=\left(\dot{u}_{1} a_{1}+u_{1} a_{13}\right) \frac{-1}{\left(R_{s}+r\right)}$

$\ddot{\gamma}=\frac{1}{R_{S}+r} \frac{\left(\dot{u}_{1} a_{2}+u_{1} a_{14}\right) S \delta+\frac{a_{2} C \delta a_{1} u_{1}^{2}}{R_{s}+r}}{S \delta^{2}}$

Now we can write equation of motion like the following state space form. State variables are defined like Eq. (46).

$x_{1}=u_{1}, x_{2}=\beta, x_{3}=\theta, x_{4}=\delta, x_{5}=\gamma$

$\left[\begin{array}{l}\dot{x}_{1} \\ \dot{x}_{2} \\ \dot{x}_{3} \\ \dot{x}_{4} \\ \dot{x}_{5}\end{array}\right]=\left[\begin{array}{l}-\frac{a_{23}}{a_{21}} x_{1}^{2}+\frac{a_{24}}{a_{21}} \\ 0 \\ \left(a_{3}-\frac{a_{2} C \delta}{\left(R_{S}+r\right) S \delta}\right) x_{1} \\ -\frac{a_{1} x_{1}}{\left(R_{S}+r\right)} \\ \frac{\left.a_{2} x_{1}\right)}{\left(R_{S}+r\right) S \delta}\end{array}\right]+\left[\begin{array}{cc}\frac{1}{R a_{21}} & -\frac{a_{22}}{a_{21} C_{b}} x_{1} \\ 0 & 1 / C_{b} \\ 0 & 0 \\ 0 & 0 \\ 0 & 0\end{array}\right]\left[\begin{array}{l}M \\ T\end{array}\right]$.

this state space equation can be shown as Eq. (23). we can find that dynamic equations of motion that they are calculated by Kane dynamic, are the same in three surfaces and the only difference is in $a_{i}$ state variables that for each of them these state variables are given in Appendix 1.

\section{Robot control algorithm}

Since the goal of this paper is controlling the robots toward the moving target and circulating it, controlling of coordinates of the robots' body center who is different in each surface of the coordinates system would be enough for controlling of the robot. Here, we define the center coordinate in all the systems like Eq. (48) that in this Eqs. (41) and (42) are different in each coordinate system that in Appendix 3 are shown for each surface.

$q=\left[\begin{array}{ll}q_{1} & q_{2}\end{array}\right]$

Controlling of the body angle based on available motion constrains cause's body angle places in a desired direction. Steering angle is controlled based on available motion constrain that in angle control section, we describe it in more details. In $[18,19]$, control of the non-holonomic systems are introduced in details, and the systems are categorized based on controllability and accessibility. In our system that a wheeler robot is considered, robot because of having wheels have nonholonomic constrains so they cannot direct to each direction toward the target simply and as a result these systems are non-controllable but because the constrains are not geometrical, robots can move to any point in the space, so these systems are accessible and there is no place limitation for them. For available non-holonomic robot, we cannot determine the velocity of the robot in a manner that $\dot{q}=\dot{q}_{d}$, nor we can get a control torque since $\ddot{q}=\ddot{q}_{d}$. Thus this system is a non-controllable system, we cannot implement feedback linearization. In this paper, using optimizing of a cost function, we can get desired robot accelerations, meanwhile using a derivative-proportional controller we can design the desired acceleration in a manner that the velocity and the position of the robot goes toward the target velocity and position. $d$ Index means desired and $t$ index means target coordinates.

$\ddot{q}_{d}=-K_{d}\left(\dot{q}-\dot{q}_{t}\right)-K_{p}\left(q-q_{t}\right)+\ddot{q}_{t}$

At last control signal determines as a function from $q, \dot{q}$ and $\ddot{q}_{d}$.

In this paper, by optimizing the norm, we will control the difference between actual and desired acceleration and since the torque of each time determines the acceleration of that time, only by optimizing this difference we can design a desired controller for the system. By parameter optimization we need fewer calculations and we will have a simple control algorithm. This procedure way is not desired for all of the non-controllable systems but it is desired for this system as we show in follow.

In (50), a cost function has been shown that must be optimized that is Euclidean norm for the difference between desired and actual acceleration.

$J=\left\|\ddot{q}-\ddot{q}_{d}\right\|$ 
If the steering angle stays in a desired direction and driving torque over front wheel has been desired, actual acceleration of the robot goes toward desired acceleration and Euclidean norm or cost function goes toward zero that in this vision it is physical and sensible. Thus based on a simple control algorithm, we can get control torques. Based on these descriptions and available cost function, Eq. (51) would be defined as follow, because $\ddot{q}_{1}$ and $\ddot{q}_{2}$ are defined in all of the coordinates separately in Appendix 3, each of them are a function from $\dot{u}_{1}, u_{1}$ and $u_{2}$.

$J^{2}=\left(\ddot{q}_{1}-\ddot{q}_{d 1}\right)^{2}+\left(\ddot{q}_{2}-\ddot{q}_{d 2}\right)^{2}$

By optimizing Eq. (51) related to acceleration of the front wheel and steering angular velocity, we can calculate control torques. First of all, we calculate desired $\dot{u}_{1}$ from Eq. (52) and then using dynamic equation of motion that is determined by Kane dynamic procedure and putting desired $\dot{u}_{1}$ in equation of motion, we can determine control torque of $M$. But desired $\dot{u}_{1}$ is a function of steering control torque. So, first we must find $T$ that its procedure is described in follow.

$\frac{\partial J^{2}}{\partial \dot{u}_{1}}=0$

To determine steering control torque, we can get it by optimizing Cost function related to steering angular velocity as Eq. (53). By using this equation, we can get desired $u_{2}$ and by placing it in dynamic equation control torque, $T$ is calculated.

$\frac{\partial J^{2}}{\partial u_{2}}=0$

But if we calculate the steering control torque like this, we would have no control on the steering angular constrain. Steering angular constrain means that this angle must not go over a special limitation or become less than it because in this state steering angular constrain breaches; thus we must use other procedure to find steering control torque, because it is supposed that steering angle can changes between $-\pi / 2$ and $\pi / 2$. Steering angle of control algorithm would be described in follow.

\subsection{Steering angle control}

Equation (54) shows desired dynamic of the steering angle that for finding it, we require to determine $\beta_{d}$ and $\dot{\beta}_{d}$ which in this equation $K_{\beta}$ is proportional controls gain. In follow we describe the procedure for finding two parameters $\beta_{d}$ and $\dot{\beta}_{d}$.

$\dot{\beta}=-K_{\beta}\left(\beta-\beta_{d}\right)+\dot{\beta}_{d}$

Based on Fig. 1 that shows the robots coordinate, constrain equations in generalized system are as follow. It should be noticed that $d$ index is desired, $E_{1}$ and $E_{2}$ indexes are for the velocity of the body center.

$v_{d}^{b}=\sqrt{v_{d_{E 1}}^{b}{ }^{2}+v_{d_{E 2}}^{b}{ }^{2}}$

$v_{d_{E 1}}^{b}=C \theta_{d} v_{d}^{b}$

$v_{d_{E 2}}^{b}=S \theta_{d} v_{d}^{b}$

$\dot{\theta}_{d}=\frac{v_{d}^{b} \tan \left(\beta_{d}\right)}{L}$

Based on Eq. (59) we can see that the motion of the robot toward the robots body angle reaches to the desired value. It should be noticed that in these equations based on the type of the mission, it would be supposed that $\dot{v}_{d_{E 1}}^{b}$ and $\dot{v}_{d_{E 2}}^{b}$ are desired accelerations for robot body center and also $v_{d_{E 1}}^{b}, v_{d_{E 2}}^{b}$ and $\ddot{v}_{d_{E 1}}^{b}, \ddot{v}_{d_{E 2}}^{b}$, can be calculated by integral and differential.

$\theta_{d}=a \tan 2\left(\frac{v_{d_{E 2}}^{b}}{v_{d}^{b}}, \frac{v_{d_{E 1}}^{b}}{v_{d}^{b}}\right)$

Now, because Eq. (60) is implemented continuously, we can by differential find the following equation.

$\dot{\theta}_{d}=\frac{\dot{v}_{d_{E 2}}^{b} v_{d_{E 1}}^{b}-\dot{v}_{d_{E 1}}^{b} v_{d_{E 2}}^{b}}{\left(v_{d}^{b}\right)^{2}}$

Using Eqs. (59) and (60), $\beta_{d}$ we will find. As it can be seen in Eq. (61), because of using $\tan ^{-1}$ the angle stays between $-\pi / 2$ and $\pi / 2$. So, steering constrain is implemented. Steering angle must follow the desired angle precisely. The existence of $\dot{\beta}_{d}$ in steering angle dynamic is because of this.

$\beta_{d}=\tan ^{-1}\left(\frac{L\left(\dot{v}_{d_{E 2}}^{b} v_{d_{E 1}}^{b}-\dot{v}_{d_{E 1}}^{b} v_{d_{E 2}}^{b}\right)}{\left(v_{d}^{b}\right)^{3}}\right)$

Now, because Eq. (61) is implemented continuously we can find the following Eq. (Fig. 5) by taking differential from $\dot{\beta}_{d}$.

$$
\begin{aligned}
\dot{\beta}_{d}=L v_{d}^{b}\left(\frac{\left(\ddot{v}_{d_{E 2}}^{b} v_{d_{E 1}}^{b}-\ddot{v}_{d_{E 1}}^{b} v_{d_{E 2}}^{b}\right)\left(v_{d}^{b}\right)^{2}}{\left(v_{d}^{b}\right)^{6}+L^{2}\left(\dot{v}_{d_{E 2}}^{b} v_{d_{E 1}}^{b}-\dot{v}_{d_{E 1}}^{b} v_{d_{E 2}}^{b}\right)}\right. \\
-\frac{3\left(\dot{v}_{d_{E 2}}^{b} v_{d_{E 1}}^{b}-\dot{v}_{d_{E 1}}^{b} v_{d_{E 2}}^{b}\right)\left(\dot{v}_{d_{E 1}}^{b} v_{d_{E 1}}^{b}-\dot{v}_{d_{E 2}}^{b} v_{d_{E 2}}^{b}\right)}{\left(v_{d}^{b}\right)^{6}+L^{2}\left(\dot{v}_{d_{E 2}}^{b} v_{d_{E 1}}^{b}-\dot{v}_{d_{E 1}}^{b} v_{d_{E 2}}^{b}\right)}
\end{aligned}
$$

Now, based on the following equation, we find the steering control torque.

$$
T=C_{b} \dot{\beta}=C_{b}\left(-K_{\beta}\left(\beta-\beta_{d}\right)+\dot{\beta}_{d}\right)
$$

Equation (63) shows the closed loop equation of error angle in which by selecting positive $K_{\beta}$, the error goes toward zero.

$\dot{e}+K_{\beta} e=0 \rightarrow e=\beta-\beta_{d}$ 


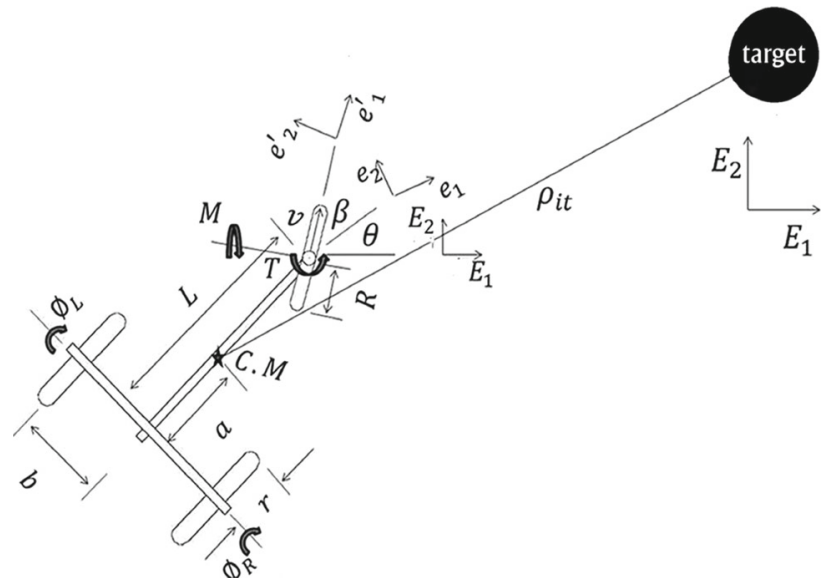

Fig. 5 Target position relative to the two following robots

Since the error dynamic equation is first order, it does not have overshoot and $\beta, \beta_{d}$ are available and the angle constrain do not breach the steering. Sometimes it is possible $\beta_{d}$ because of $\tan ^{-1}$ in its calculation discontinuously changes from $\pm \pi / 2$ to $\mp \pi / 2$ that it is because of filter (63). Sometimes it is required that control torque becomes very big and in this situation the actuator can only get maximum external torque. Thus we must consider the effect of the saturation and filtering of actuators that this work is introduced by these two functions. In these equations, $u$ is the ideal torque signal and $\tau$ is the output of the actual actuator and $\rho$ is reverse pole of the filter that by its extending, the ability of the actuator for following the step input reduces.

$$
\begin{aligned}
& \tau=\left\{\begin{array}{ll}
u & |u|<\tau_{\max } \\
\tau_{\max } \operatorname{sign}(u) & |u|>\tau_{\max }
\end{array}\right\} \\
& \tau(s)=\frac{u(s)}{\rho s+1}
\end{aligned}
$$

\section{Mobile target dynamic}

Mobile target is supposed like a paper with mass that has holonomic dynamic. Therefore, it can move to each direction without any geometrical constrain

We suppose the target dynamic in a manner that by coming close to the robot, the target escapes until there would be a read state for it. Equation (67) shows the mobile target equation of motion that in this equation $m_{t}$ is the target's mass and $K_{t}$ is the target escape force coefficient and $C_{t}$ is damping coefficient. Index $t$ shows the targets coordinate.

$m_{t}\left[\begin{array}{l}\ddot{q}_{E 1}^{t} \\ \ddot{q}_{E 2}^{t}\end{array}\right]=K_{t} F_{t}-C_{t}\left[\begin{array}{l}\dot{q}_{E 1}^{t} \\ \dot{q}_{E 2}^{t}\end{array}\right]$

In this equation $F_{t}$ is the target's escape force. The targets damping force is a factor that prevents from extending of the target's velocity.

$$
\begin{aligned}
& F_{t}=\sum_{i=1}^{n} \frac{\overrightarrow{e_{i t}}}{\left\|\overrightarrow{\rho_{i t}}\right\|}=\sum_{i=1}^{n} \frac{\overrightarrow{\rho_{i t}}}{\left\|\overrightarrow{\rho_{i t}}\right\|^{2}} . \\
& \left\|\overrightarrow{\rho_{i t}}\right\|=R_{i t}
\end{aligned}
$$

In Eq. (68), $\vec{\rho}_{i t}$ is the relative position vector between robots and the target that based on the kind of surface and coordinate system is different. Generalized coordinates for robots in each surface are shown in Appendix 3 that they are the same for the target.

\section{Designing of controller for the set of robots}

The purpose of this section is getting desired accelerations $\left(\ddot{q}_{1 d}, \ddot{q}_{2 d}\right)$.

The purpose of the paper is controlling a set of wheeler robots that have mass. In this mission maybe robots stay around the target or by making a cover in its target cause making a supportive mission. Thus, the purpose is following the target and placing in an equilibrium distance from it. In [20] we have used following analyze for implementing group mission and with a few changes on this algorithm; we do the group mission of this paper.

Based on Fig. 6 we can see that in this paper it is supposed that the spring and dampers are available between the robots and the target and as we can see in this figure $K_{S}$ shows spring coefficient, $C_{L}$ and $C_{R}$ are damping coefficients. This set has two robots and one target that all of them have masses. Based on these descriptions and Fig. 6 we will get a better vision. Desired acceleration for each robot is designed using accelerations in a manner that none of the robots and the target, accident with each other. Equilibrium distance of the robots is determined by their dimensions. If we say that the desired acceleration is designed based on other robots accelerations, it does not mean that other robots accelerations are desired in acceleration equation. In fact putting more acceleration in the equation of desired acceleration is meaningless but we will bring the target accelerations in the desired acceleration equation. In Eqs. (69) and (70), the target acceleration

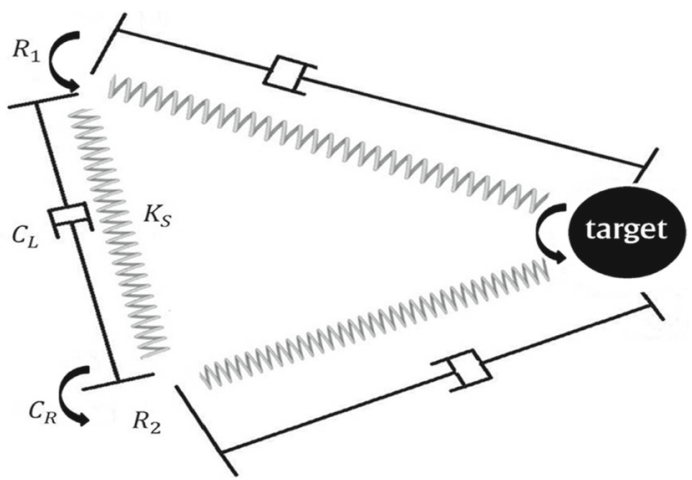

Fig. 6 Physical descriptions of the control algorithm stated by mechanical elements 
and robots desired acceleration $i$ are introduced that in these equations, $q$ is generalized coordinates in all of the surfaces that is defined in Appendix 3.

$$
\begin{aligned}
& a_{t}=\left[\begin{array}{ll}
\ddot{q}_{E 1}^{t} & \ddot{q}_{E 2}^{t}
\end{array}\right]^{T} \\
& \ddot{q}_{d i}=\left[\begin{array}{ll}
\ddot{q}_{E 1}^{d i} & \ddot{q}_{E 2}^{d i}
\end{array}\right]^{T}
\end{aligned}
$$

In Eq. (71), relative distance and its size between two robots and the robot and the target are introduced.

$$
\begin{array}{ll}
r_{i j}=\left[\begin{array}{ll}
q_{E 1}^{j}-q_{E 1}^{i} & q_{E 2}^{j}-q_{E 2}^{i}
\end{array}\right]^{T}, \quad R_{i j}=\left\|r_{i j}\right\| \\
r_{i t}=\left[\begin{array}{ll}
q_{E 1}^{t}-q_{E 1}^{i} & q_{E 2}^{t}-q_{E 2}^{i}
\end{array}\right]^{T}, \quad R_{i t}=\left\|r_{i t}\right\|
\end{array}
$$

Finally in (71), we define the desired acceleration of robot $i$. In this equation $R_{i j}$ is the distance between two robots and $R_{i t}$ is the distance between robot and the target. Meanwhile $R_{R}$ is equilibrium distance between the robots that it is determined based on the robot sizes. $R_{t}$ is equilibrium distance between the robots and the target. $K_{d}$ and $K_{p}$ are derivative and proportional control coefficients respectively that they are for reduction of velocity between robots and setting the distance between them. $K_{d}^{t}$ and $K_{p}^{t}$ are derivative and proportional control coefficients between the robot and the target.

$$
\begin{aligned}
\ddot{q}_{d i}= & \sum_{\substack{j=1 \\
j \neq i}}^{n}\left(K_{p}\left(R_{i j}-R_{R}\right) \frac{r_{i j}}{R_{i j}}+K_{d} \dot{r}_{i j}\right) \\
& +K_{p}^{t}\left(R_{i t}-R_{t}\right) \frac{r_{i t}}{R_{i t}}+K_{d}^{t} \dot{r}_{i t}+a_{t}
\end{aligned}
$$

Equilibrium distance is determined based on the kind of the maneuver and the insurance limitation between robots, unit no accident occurs. For example, robots can completely make a circle around the target if equilibrium distance is in a manner that the target stays in the center of the robots. Based on the purpose of this paper, equilibrium distance between robots and the target is important as a result proportional controller coefficient must be large enough but its largeness causes that more acceleration enters on the robot and chaos occurs. Therefore, constant control coefficient is not suitable for this mission and we must use variable coefficients. This mission for change of gain is done by a math function and automatically.

$$
\begin{aligned}
& \mu=R_{i t}-\left(R_{t}+\kappa\right) \\
& \chi=\frac{1+\tanh \left(\mu \times \varepsilon^{2}\right)}{2} \\
& {\left[\begin{array}{ll}
K_{p}^{t} & K_{d}^{t}
\end{array}\right]^{T}=\chi\left[\begin{array}{ll}
K_{p 1}^{t} & K_{d 1}^{t}
\end{array}\right]^{T}} \\
& +(1-\chi)\left[K_{p 2}^{t} K_{d 2}^{t}\right]^{T}
\end{aligned}
$$

In (73), $\kappa$ shows in which distance from equilibrium distance, coefficient change. And in (74), $\chi$ is a coefficient between zero and one. If the distance between robot and the target is more than equilibrium distance, this coefficient is one and if it is less than equilibrium distance, this coefficient is zero. In (74), $\varepsilon$ is for increasing of $\chi$ changes. It should be noticed that during the mission, derivative control must be continuously done among the robots until controls the velocity between them. But there are no needs that implement the proportional control between them continuously. When relative distance of them is less than equilibrium distance, proportional control enters and prevents from their accident and if robots reach to the equilibrium distance, the mission completes.

\section{Estimator design}

To design the estimator, we introduce the suppositions related to measurements. In this paper it is supposed that the mobile target knows the place of each robot exactly and based on it, calculates the force of robots precisely. It is supposed about the robots that all of them have exact sensors those asses the body angle and steering's angle and front wheels velocity. Meanwhile, robots have wireless antennas for receiving signal. There are land marks that show exact places of them. Robots using them find their places that this act is called localization. Based on control algorithm we can get control torques but they produce some noises. As we see in following, this noise is Gaussian and we get suitable results. Robot target their information like steering angle, front wheel velocity and the body angle to other robots. Because the goal is implementing a group mission, robots must have information about relative position and other robots and target relative velocity. If robots can use (GPS), using velocity and absolute position they can find velocity and relative position. But this procedure may have limitation. Thus in this paper it is supposed that a flying device using image processing, sends the relative position of robots and the target as well as noise to the estimator robot.

\subsection{Estimating relative state variables of robots using estimator robot}

As it can be seen in Fig. 7, robots state variables $i$ are estimated by estimator robot $j$. robot $j$ based on assessed information using flying device and sent information from robot $i$ that receives by antenna and wireless communication, estimates the relative distance and robots state variables $i$.In this paper, extended Kalman filter and then extended Kalman smoother are used for estimation.

In Fig. 7, $\rho_{i j}$ is relative distance between two robots and $\vartheta_{i j}$ is relative angle between two robots that is assessed by flying device and is sent to estimator robot. Robot $i$ send its information to the estimator robot and this robot uses these information and Kalman estimators, estimates robot $i$ state 


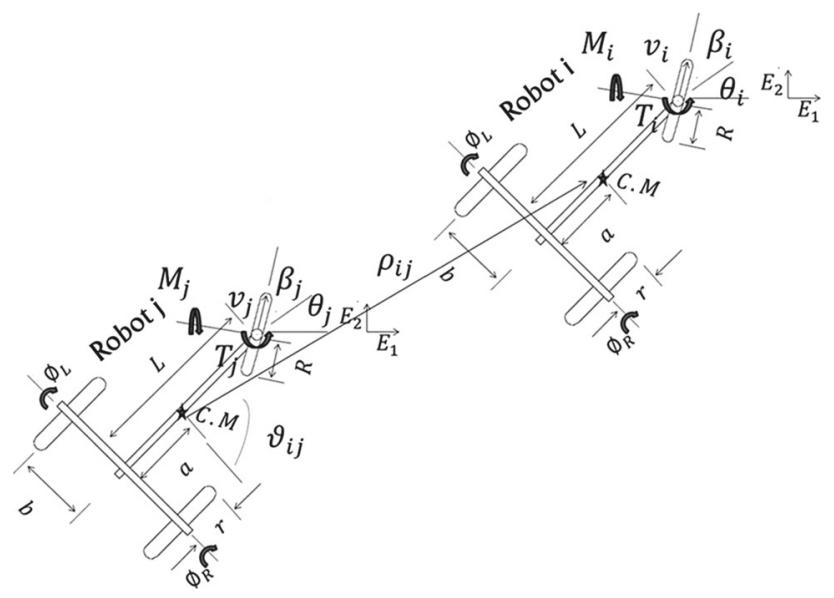

Fig. 7 Schematic diagram showing signal transmission between transmitter robot $i$ and receiving robot $j$

variables. In follow, relative equations of motion between robots $i$ and $j$ are shown and movement constrains and dynamic equation is used to get relative equation of motion. In the following equations, $q_{1}$ and $q_{2}$ are generalized coordinates of robot. It should be noticed that some of the state variables of the robot $j$ are not obvious that the way for their estimation is introduced in the next section.

$q_{1}^{i j}=q_{1}^{i}-q_{1}^{j}$

$q_{2}^{i j}=q_{2}^{i}-q_{2}^{j}$

$\vartheta_{i j}=\frac{\pi}{2}+a \tan 2\left(q_{2}^{i j}, q_{1}^{i j}\right)-\theta_{j}$

$\alpha_{i j}=\vartheta_{i j}+\theta_{j}-\theta_{i}$

$\rho_{i j}=\sqrt{\left(q_{2}^{i j}\right)^{2}+\left(q_{1}^{i j}\right)^{2}}$

$\dot{\rho}_{i j}=\frac{\dot{q}_{1}^{i j} q_{1}^{i j}-\dot{q}_{2}^{i j} q_{2}^{i j}}{\rho_{i j}}=v_{i} S \alpha_{i j}-v_{j} S \vartheta_{i j}$

$\dot{\vartheta}_{i j}=\frac{\dot{q}_{2}^{i j} q_{1}^{i j}-\dot{q}_{1}^{i j} q_{2}^{i j}}{\rho_{i j}^{2}}-\dot{\theta}_{j}=\frac{v_{i} C \alpha_{i j}-v_{j} C \vartheta_{i j}}{\rho_{i j}}-\dot{\theta}_{j}$

Using (81) and (82) and equation of motion of each robot that estimated for each surface, we will find the relative equation of motion between two robots $(i$ and $j$ ). In Appendix 4 , relative equation of motion related to each surface is get separately. Relative equation of motion can be getting like (83).

$\dot{X}_{i j}=f\left(X_{i j}, \tau\right)+w$

That in this equation, state variables are as follow.

$x_{1}=u_{1}^{i}, x_{2}=\beta_{i}, x_{3}=\theta_{i}, x_{4}=\rho_{i j}, x_{5}=\vartheta_{i j}$
In this equation, $\tau$ is control torque of the two robots ( $i$ and $j), w$ is also noise on the model. Robot $j$ based on its sensors, calculates its control torque exactly but because it does not know position and velocity of the robot $i$, exactly and only estimated it, robot is control torques can't be calculated correctly, as a result $\widetilde{T}_{i}$ with $T_{i}$ and $\widetilde{M}_{i}$ with $M_{i}$ are different and they have a relation based on this fact.

$$
\left[\begin{array}{ll}
\widetilde{T}_{i} & \widetilde{M}_{i}
\end{array}\right]^{T}=\left[\begin{array}{ll}
T_{i} & M_{i}
\end{array}\right]^{T}+\tau_{\text {error }}^{i}
$$

As it is shown in (85), $\widetilde{\tau}_{i}$ like $\tau_{\text {error }}$ has difference with $\tau_{i}$. Torque distribution of $\tau_{\text {error }}^{i}$ is not obvious and no model can be specified to it. Thus, this error appears like a noise in the system. Its effect on state variables has an effect like $\tau_{\text {error }}^{i}$. This noise is calculated based on Eq. (84). In this equation, $Q_{C}^{i}$ is the noise power.

$\tau_{\text {error }}^{i}=N\left(0, Q_{C}^{i}\right) \rightarrow Q_{C}^{i}=\left[\begin{array}{cc}\left(\sigma_{M}^{i}\right) & 0 \\ 0 & \left(\sigma_{T}^{i}\right)\end{array}\right]$

Noise covariance matrix is defined like follow:

$Q^{i}(K)=B^{i} Q_{C}^{i}\left(Q_{C}^{i}\right)^{T}\left(B^{i}\right)^{T} d t$

In this equation $a_{k}$ and $a_{n}$ are different based on the kind of the surface. In ramp surface, $a_{9}$ and $a_{10}$ and in cylindrical surface $a_{17}$ and $a_{18}$ and in spherical surface $a_{21}$ and $a_{22}$. It should be noticed that in (83), there is an equation with continuous time that we need for using in Kalman filter and we must change it to discrete time system. For discretion different ways are used.

Available methods include Euler method, trapezoidal method and Runge-Kutta method. Because we use these methods for estimation and only for Kalman filter and smoother are used, Euler method is suitable and in estimator this error corrects repeatedly. In follow, discreted form of (83) is introduced by Euler method. Equation (88) has been used only for estimation but this model can be getting by a more precise method. To study discretization methods refer to $[21]$.

$$
\begin{aligned}
X_{i j}(K)= & \left(f\left(X_{i j}(K-1)\right), \tau(K-1)\right) d t \\
& +X_{i j}(K-1)+w(K-1)
\end{aligned}
$$

In Eq. (88) dt is time step in discretization. In following, we introduce the output of the measurements. As it was mentioned the first measurement is a flying device that reports the relative angle and distances that estimator gets and sends this information that is with noise and the noise introduces as available noise in sensor.

$Z_{1}^{i j}=\left[\begin{array}{c}\rho_{i j}+v_{\rho}^{i j} \\ \vartheta_{i j}+v_{\vartheta}^{i j}\end{array}\right]=\left[\begin{array}{c}\rho_{i j} \\ \vartheta_{i j}\end{array}\right]+v_{1}^{i j}$

In this equation $v_{\rho}^{i j}$ and $v_{\vartheta}^{i j}$ are available noises in sensors and are introduced as Eq. (90). In this equation $R_{1}^{i j}(K)$ is 
power spectral density Noise matrix.

$v_{1}^{i j}=N\left(0, R_{1}^{i j}(K)\right)$

$R_{1}^{i j}(K)=\left[\begin{array}{ll}\left(\sigma_{\rho}^{i j}\right)^{2} & 0 \\ 0 & \left(\sigma_{\vartheta}^{i j}\right)^{2}\end{array}\right]$

For this sensor, we will get output matrix using Taylor approximation by (91). Output matrix can be getting by (92).

$$
\begin{array}{r}
{\left[H_{x}\left(m_{k}\right)\right]_{j, j^{\prime}}=\frac{\partial h_{j}\left(x_{k}\right)}{\partial x_{j^{\prime}}} x=m} \\
H_{1}^{i j}=\left[\begin{array}{lllll}
0 & 0 & 0 & 1 & 0 \\
0 & 0 & 0 & 0 & 1
\end{array}\right]
\end{array}
$$

As it was mentioned, there is a second sensor that measured the velocity of front wheel, steering angle and robot body angle and sends this information for estimator robot that we have the following equation.

$Z_{2}^{i j}=\left[\begin{array}{c}u_{1}^{i}+v_{u_{1}}^{i} \\ \beta_{i}+v_{\beta}^{i} \\ \theta_{i}+v_{\theta}^{i}\end{array}\right]=\left[\begin{array}{c}u_{1}^{i} \\ \beta_{i} \\ \theta_{i}\end{array}\right]+v_{2}^{i j}$

$v_{2}^{i j}$ Is the noise of measurement and like first sensor's noise is considered as Gaussian noise.

$$
\begin{aligned}
& v_{2}^{i j}=N\left(0, R_{2}^{i j}(K)\right) \\
& R_{2}^{i j}(K)=\left[\begin{array}{lll}
\left(\sigma_{u_{1}}^{i j}\right)^{2} & 0 & 0 \\
0 & \left(\sigma_{\beta}^{i j}\right)^{2} & 0 \\
0 & 0 & \left(\sigma_{\theta}^{i j}\right)^{2}
\end{array}\right]
\end{aligned}
$$

Output matrix becomes like Eq. (96).

$$
H_{2}^{i j}=\left[\begin{array}{lllll}
1 & 0 & 0 & 0 & 0 \\
0 & 1 & 0 & 0 & 0 \\
0 & 0 & 1 & 0 & 0
\end{array}\right]
$$

Total output matrix as well as total noise covariance matrix is introduced by following equations.

$$
\begin{aligned}
R^{i j} & =\left[\begin{array}{ll}
R_{1}^{i j}(K) & 0_{2 \times 3} \\
0_{3 \times 2} & R_{2}^{i j}(K)
\end{array}\right] \\
H^{i j} & =\left[\begin{array}{l}
H_{1}^{i j} \\
H_{2}^{i j}
\end{array}\right] \\
Z^{i j} & =\left[\begin{array}{l}
Z_{1}^{i j} \\
Z_{2}^{i j}
\end{array}\right]
\end{aligned}
$$

To implement extended Kalman filter algorithm we need to estimate the output that is introduced like the following equation.
$\widetilde{Z}^{i j}=\left[\begin{array}{l}H_{1}^{i j} \\ H_{2}^{i j}\end{array}\right] \widetilde{X}^{i j}$

State transition matrix will be getting by Taylor's approximation equation (101).

$\left[F^{i j}\right]_{k, k^{\prime}}=\frac{\partial f_{k}}{\partial x_{k^{\prime}}} d t+I_{5 \times 5}$

Based on calculation of required parameters in extended Kalman filter in previous, now using extended Kalman algorithm, we can estimate state variables of relative equation of motion. Extended Kalman filter algorithm has been shown in Eq. (102) in two step time. By estimating state variables using Kalman filter, we can place estimated variables once again in extended Kalman smoother algorithms (103) to get better estimation. The difference between these two estimators is discussed in conclusion. It should be noticed that in this section, Matlab functions of [22] are used.

\section{Predict}

$m_{k}^{-}=f\left(m_{k-1}, u_{k-1}\right)$

$P_{k}^{-}=F_{x}\left(m_{k-1}, u_{k-1}\right) P_{k-1} F_{x}^{T}\left(m_{k-1}, u_{k-1}\right)+Q_{k-1}$

Update

$$
\begin{aligned}
& V_{k}=y_{k}-h\left(m_{k}^{-}\right) \\
& S_{k}=H_{x}\left(m_{k}^{-}\right) P_{k}^{-} H_{x}^{T}\left(m_{k}^{-}\right)+R_{k} \\
& K_{k}=P_{k}^{-} H_{x}^{T}\left(m_{k}^{-}\right) S_{k}^{-1} \\
& m_{k}=m_{k}^{-}+K_{k} V_{k} \\
& P_{k}=P_{k}^{-}-K_{k} S_{k} K_{k}^{T}
\end{aligned}
$$

In this equation $m_{k}^{-}$and $P_{k}^{-}$are mean estimation and state variables covariance in time $\mathrm{k}$ before seeing measurement. $P_{k}$ and $m_{k}$ are mean estimation and state variable covariance in time $k$ after seeing measurement. $S_{k}$ shows measurement of covariance estimation in time $\mathrm{k}$ and $K_{k}$ is gain of the filter that shows how the estimation reforms in time $k$ [22-24].

$$
\begin{aligned}
m_{k+1}^{-} & =f\left(m_{k}, u_{k}\right) \\
P_{k+1}^{-} & =F_{x}\left(m_{k}, u_{k}\right) P_{k} F_{x}^{T}\left(m_{k}, u_{k}\right)+Q_{k} \\
C_{k} & =P_{k} F_{x}^{T}\left(m_{k}, u_{k}\right)\left[P_{k+1}^{-}\right]^{-1} \\
m_{k}^{s} & =m_{k}+C_{k}\left[m_{k+1}^{s}-m_{k+1}^{-}\right] \\
P_{k}^{s} & =P_{k}+C_{k}\left[P_{k+1}^{s}-P_{k+1}^{-}\right] C_{k}^{T}
\end{aligned}
$$

$P_{k}^{s}$ and $m_{k}^{s}$ are mean estimation and state covariance in step time of $k$ by the smoother. $C_{k}$ is gain of the smoother that shows how estimation smoother reforms.

\subsection{Localization}

To estimate robot state variables it is supposed that based on available sensors on the robot, robot body angle, steering angle and front wheel velocity are recognized exactly and the variables that must be estimated is Place the center of the 
robot in each of the surfaces. As it was mentioned in each of the surfaces, there are a number of landmarks that their exact place is obvious. Using sensors on the robot, it finds its relative place in respect to them and gets information about its place. Robot equation of motion in each of the surfaces was introduced in previous sections. State transition matrix can be accessed by following equation.

$$
\left[F^{i P}\right]_{k, k^{\prime}}=\frac{\partial f_{k}}{\partial x_{k^{\prime}}} d t+I_{5 \times 5}
$$

The place of landmarks is like $P_{i}=\left(P_{q_{1}}, P_{q_{2}}\right)$. Output function of sensor is like Eq. (107). In following equations $\rho_{i}^{P_{k}}$ is the distance of robot $i$ with $P_{k}$ Landmark and $\vartheta_{i}^{P_{k}}$ is vision angle of $P_{k}$ Landmark by robot $i$.

$\rho_{i}^{P_{k}}=\sqrt{\left(P_{q_{2}}-q_{2}^{i}\right)^{2}+\left(P_{q_{1}}-q_{1}^{i}\right)^{2}}$

$\vartheta_{i}^{P_{k}}=a \tan 2\left(P_{q_{2}}-q_{2}^{i}, P_{q_{1}}-q_{1}^{i}\right)-\theta_{i}$

$Z_{i}^{P_{k}}=\left[\begin{array}{c}\rho_{i}^{P_{k}}+v_{\rho_{i}}^{P_{k}} \\ \vartheta_{i}^{P_{k}}+v_{\vartheta_{i}}^{P_{k}}\end{array}\right]=\left[\begin{array}{c}\rho_{i}^{P_{k}} \\ \vartheta_{i}^{P_{k}}\end{array}\right]+v_{i}^{P_{k}}$

In this equation $v_{\rho_{i}}^{P_{k}}$ and $v_{\vartheta_{i}}^{P_{k}}$ are available noise in sensor and are introduced like (108). In this equation $R_{1}^{P_{k}}(K)$ is power spectral density noise matrix.

$v_{i}^{P_{k}}=N\left(0, R_{i}^{P_{k}}(K)\right)$

$R_{i}^{P_{k}}(K)=\left[\begin{array}{ll}\left(\sigma_{\rho_{i}}^{P_{k}}\right)^{2} & 0 \\ 0 & \left(\sigma_{\vartheta_{i}}^{P_{k}}\right)^{2}\end{array}\right]$

Output matrix can be accessed like Eq. (109).

$$
\begin{aligned}
& H_{i}^{P_{k}}=\frac{1}{\left(r_{P}\right)^{2}}
\end{aligned}
$$

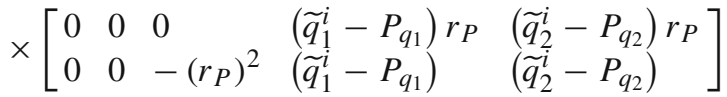

$r_{p}$ is the distance between robot $i$ and $P_{k}$ Landmark. Now, using extended Kalman filter we can estimate the place of the robot.

\subsection{Estimating robot relative state variables and the target}

In this section we estimate the target state variables by robot estimator. As it can be seen in Fig. 8, the target state variables are estimated by estimator robot of $i$. Robot $i$ estimates relative distance and the target state variables by flying device and sent data from the target that receives by antenna and wireless communication. In this paper, Kalman optimized filter and then Kalman optimized smoother are used for estimation.

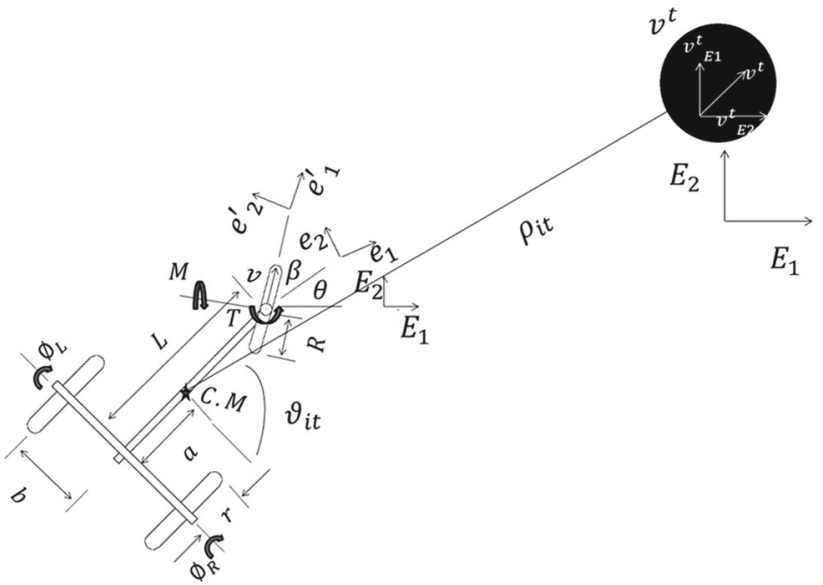

Fig. 8 Schematic diagram showing range and bearing between robot $i$ and the target

In Fig. $8, \rho_{i t}$ is relative distance between the robot and the target and $\vartheta_{i t}$ it the relative angle between these two that flying device measured it and sends to estimator robot and the target sends its information for estimator robot and the estimator robot uses this information and using Kalman estimators estimates the target state variables.

In the following, relative equations of motion between robots $i$ and the target are shown and movement constrains and dynamic equation is used to get relative equation of motion.

$q_{1}^{t i}=q_{1}^{t}-q_{1}^{i}$

$q_{2}^{t i}=q_{2}^{t}-q_{2}^{i}$

$\vartheta_{t}=a \tan 2\left(\dot{q}_{2}^{t}, \dot{q}_{1}^{t}\right)$

$v_{t}=\sqrt{\left(\dot{q}_{2}^{t}\right)^{2}+\left(\dot{q}_{1}^{t}\right)^{2}}$

$\vartheta_{t i}=\frac{\pi}{2}+a \tan 2\left(q_{2}^{t i}, q_{1}^{t i}\right)-\theta_{i}$

$\alpha_{t i}=\vartheta_{t i}+\theta_{i}-\theta_{t}$

$\rho_{t i}=\sqrt{\left(q_{2}^{t i}\right)^{2}+\left(q_{1}^{t i}\right)^{2}}$

$\dot{\rho}_{t i}=\frac{\dot{q}_{1}^{t i} q_{1}^{t i}-\dot{q}_{2}^{t i} q_{2}^{t i}}{\rho_{t i}}=v_{t} S \alpha_{t i}-v_{i} S \vartheta_{t i}$

$\dot{\vartheta}_{t i}=\frac{\dot{q}_{2}^{t i} q_{1}^{t i}-\dot{q}_{1}^{t i} q_{2}^{t i}}{\rho_{t i}^{2}}-\dot{\theta}_{i}=\frac{v_{t} C \alpha_{t i}-v_{i} C \vartheta_{t i}}{\rho_{t i}}-\dot{\theta}_{i}$

Using Eqs. (117) and (118) and also the target equation of motion that calculated in previous sections for each of the surfaces separately, we would calculate the relative equation of the motion between the robot $i$ and the target. In Appendix 5 , relative equation of motion related to each of the surfaces is introduced separately. In total, relative equation of motion is like Eq. (119).

$\dot{X}_{t i}=f\left(X_{t i}, \tau\right)+w$ 
In this equation, $F$ is the target escaping force. $w$ is the noise of the model. As it was mentioned, robot $i$ using its sensors, calculates its control torque exactly but since it does not know the target escaping force by error and by noise, as a result is different $\widetilde{F}_{t}$ with $F_{t}$ and their relation is as follows.

$F_{t}=\widetilde{F}_{t}+F_{\text {error }}^{t}$

As it is shown in (120), $\widetilde{F}_{t}$ is different from $F_{t}$ like $F_{\text {error }}$. Force distribution of $F_{\text {error }}^{t}$ is completely unknown and no model can be related to it. Thus, this error appears like a noise in the model that we estimate this error like a Gaussian noise by zero mean that it effect on state variables is like $F_{\text {error }}^{t}$ noise on the model is calculated like Eq. (121). In this equation, $Q_{C}^{t}$ is the noise power.

$F_{\text {error }}^{t}=N\left(0, Q_{C}^{t}\right) \rightarrow Q_{C}^{t}=\left[\begin{array}{ll}\left(\sigma_{F_{E 1}}^{t}\right) & 0 \\ 0 & \left(\sigma_{F_{E 2}}^{t}\right)\end{array}\right]$

The noise covariance is defined as follows. In this equation $B^{t}$ matrix is different in each surface and it is shown in Appendix 5.

$Q^{i}(K)=B^{t} Q_{C}^{t}\left(Q_{C}^{t}\right)^{T}\left(B^{t}\right)^{T} d t$

What should be mentioned is that Eq. (119) is a continuous equation with time that we need change it to a discrete system by time for using in Kalman filter.

Discrete form Eq. (119) is shown in the following like rectangular method. Equation (123) is used only for estimation.

$X_{t i}(K)=\left(f\left(X_{t i}(K-1)\right), \tau(K-1)\right) d t+$

$X_{t i}(K-1)+w(K-1)$

Following, we introduce the output of sensors. As it was mentioned the first sensor is a flying device that reports the estimator robot relative angle and distance with a target that its state variables must be estimated that this sending of information has noise and as we will see since we cannot find a model for this noise, we consider it as a white Gaussian noise which this noise is considered as available noise in sensor.

$Z_{1}^{t i}=\left[\begin{array}{c}\rho_{t i}+v_{\rho}^{t i} \\ \vartheta_{t i}+v_{\vartheta}^{t i}\end{array}\right]=\left[\begin{array}{c}\rho_{t i} \\ \vartheta_{t i}\end{array}\right]+v_{1}^{t i}$

In this equation $v_{\rho}^{t i}$ And $v_{\vartheta}^{t i}$ Are considered as available noises in sensor and are redefined as Eq. (125). In this equation $R_{1}^{t i}(K)$ is noise power of the matrix.

$v_{1}^{t i}=N\left(0, R_{1}^{t i}(K)\right)$

$R_{1}^{t i}(K)=\left[\begin{array}{ll}\left(\sigma_{\rho}^{t i}\right)^{2} & 0 \\ 0 & \left(\sigma_{\vartheta}^{t i}\right)^{2}\end{array}\right]$

Output matrix is obtained like Eq. (126).
$H_{1}^{t i}=\left[\begin{array}{llll}0 & 0 & 1 & 0 \\ 0 & 0 & 0 & 1\end{array}\right]$

As it was mentioned there is a second sensor that measurments the target velocity in each direction and sends for the estimator robot that we introduce it as following equation.

$Z_{2}^{t i}=\left[\begin{array}{c}\dot{q}_{2}^{t}+v_{\dot{q}_{2}}^{t} \\ \dot{q}_{1}^{t}+v_{\dot{q}_{1}}^{t}\end{array}\right]=\left[\begin{array}{c}\dot{q}_{2}^{t} \\ \dot{q}_{1}^{t}\end{array}\right]+v_{2}^{t i}$

$v_{2}^{t i}$ Is noise of the measurement and like noise of the first sensor is regarded as a Gaussian noise.

$v_{2}^{t i}=N\left(0, R_{2}^{t i}(K)\right)$

$R_{2}^{t i}(K)=\left[\begin{array}{cc}\left(\sigma_{\dot{q}_{2}}^{t i}\right)^{2} & 0 \\ 0 & \left(\sigma_{\dot{q}_{1}}^{t i}\right)^{2}\end{array}\right]$

Output matrix is accessed like Eq. (129).

$H_{2}^{t i}=\left[\begin{array}{llll}1 & 0 & 0 & 0 \\ 0 & 1 & 0 & 0\end{array}\right]$

Total output matrix and total noise covariance matrix are as following equations.

$$
\begin{aligned}
R^{t i} & =\left[\begin{array}{ll}
R_{1}^{t i}(K) & 0_{2 \times 2} \\
0_{2 \times 2} & R_{2}^{t i}(K)
\end{array}\right] \\
H^{t i} & =\left[\begin{array}{l}
H_{1}^{t i} \\
H_{2}^{t i}
\end{array}\right] \\
Z^{t i} & =\left[\begin{array}{l}
Z_{1}^{t i} \\
Z_{2}^{t i}
\end{array}\right]
\end{aligned}
$$

To implement extended Kalman filter algorithm we require to estimate the output that is as follow.

$\widetilde{Z}^{t i}=\left[\begin{array}{c}H_{1}^{t i} \\ H_{2}^{t i}\end{array}\right] \widetilde{X}^{t i}$

State transition matrix can be accessed by Eq. (134).

$\left[F^{t i}\right]_{k, k^{\prime}}=\frac{\partial f_{k}}{\partial x_{k^{\prime}}} d t+I_{5 \times 5}$

Based on required calculated parameters in extended Kalman filter, in this section using extended Kalman algorithm we can estimate state variables of relative equation of motion. By estimating state variables using Kalman filter we can replace again estimated variables in Kalman smoother algorithm for better estimation.

\section{Simulation results}

in this section using control algorithms, the four robots are controlled in a manner to hunt the required target or make a cover around it. Required algorithms are used for three surfaces and their diagrams based on relative distances and velocity and also control torques and the steering angles 
Table 1 Simulations parameters

\begin{tabular}{|c|c|}
\hline$d t=0.05 \mathrm{~s}$ & Discretization step time \\
\hline $\begin{array}{l}m_{b}=5(\mathrm{Kg}), m_{w}=0.4(\mathrm{Kg}), L=2(\mathrm{~m}), a=0, \\
b=0.75(\mathrm{~m}), R=0.6(\mathrm{~m}), r=0.1(\mathrm{~m})\end{array}$ & Robot mass and geometries \\
\hline$\rho=0.3, M_{\max }=15(\mathrm{Nm}), T_{\max }=1.5(\mathrm{Nm})$ & Actuator parameters \\
\hline$K_{d}=1, K_{p}=5$ & $\begin{array}{l}\text { Damping and spring (repulsive) coefficient between } \\
\text { robots }\end{array}$ \\
\hline$K_{d}^{t}=4, K_{p}^{t}=0.5$ & $\begin{array}{l}\text { Damping and spring coefficient between robots and } \\
\text { target at long distances }\end{array}$ \\
\hline$K_{d}^{t}=3, K_{p}^{t}=3$ & $\begin{array}{l}\text { Damping and spring coefficient between robots and } \\
\text { target at small distances }\end{array}$ \\
\hline$\kappa=2, \varepsilon=2$ & Parameters of controller coefficient variations \\
\hline$K_{\beta}=1, C_{b}=1$ & Coefficient of steering controller and damping \\
\hline$m_{t}=1, K_{t}=1, C_{t}=1$ & Mass, damping and coefficient of target escape force \\
\hline$R_{t}=5(\mathrm{~m})$ & Equilibrium distance between robot and target \\
\hline$R_{R}=5 \sqrt{2}(\mathrm{~m})$ & $\begin{array}{l}\text { Equilibrium distance between two robots during } \\
\text { hunting the target }\end{array}$ \\
\hline$R_{R}=0.7 \times 5 \sqrt{2}(\mathrm{~m})$ & $\begin{array}{l}\text { Equilibrium distance between two robots during } \\
\text { covering around the target }\end{array}$ \\
\hline$\sigma_{M}^{i}=0.05, \sigma_{T}^{i}=0.05$ & Applied noise to robot (i) dynamics \\
\hline$\sigma_{u_{1}}^{i j}=\sigma_{\theta}^{i j}=\sigma_{\beta}^{i j}=0.01$ & Added noise to received data sent by robot \\
\hline$\sigma_{\rho}^{i j}=\sigma_{\rho}^{i t}=0.2, \sigma_{\vartheta}^{i j}=\sigma_{\vartheta}^{i t}=0.05$ & Added noise to tracer (flying equipment) data \\
\hline$\sigma_{\rho_{i}}^{P_{k}}=0.1, \sigma_{\vartheta_{i}}^{P_{k}}=0.03$ & $\begin{array}{l}\text { Added noise to Landmarks distance and view angle } \\
\text { sensor }\end{array}$ \\
\hline$\sigma_{F}^{t}=0.05$ & Applied noise to target dynamics \\
\hline$\sigma_{\dot{q}}^{t i}=0.01$ & Added noise to target velocity sensor \\
\hline
\end{tabular}

would be accessed in all of the surfaces. Meanwhile, estimation caused by filter and smoother are introduced and a comparison is introduced. Used parameters are shown in Table 1.

Equilibrium distance between robots and the target is considered as $R_{t}=5$ and since there are four robots, during hunting the target, the distance between robots is calculated based on $R_{R}=5 \sqrt{2}$. For following mission, robots must come close to each other, thus their distance is calculated by $R_{R}=0.7 \times 5 \sqrt{2}$.

\subsection{Hunting missions target at three surfaces}

In this mission four robots are used that all of them were expressed as a constraint and limitation of motion that they are non-holonomic. But required target is considered as a mass point and is holonomic and it is considered that the target is escaping. The mission is in a manner that robots make a circle around the target and hunt it and the target velocity reaches to zero. Sometimes the target escapes and an imbalance occurs.

As, it is seen in Fig. 9 the target is hunted by four robots and the target's final is shown by a square. The implementation of mission is not based on prior arrangement of robots as we see in follow.

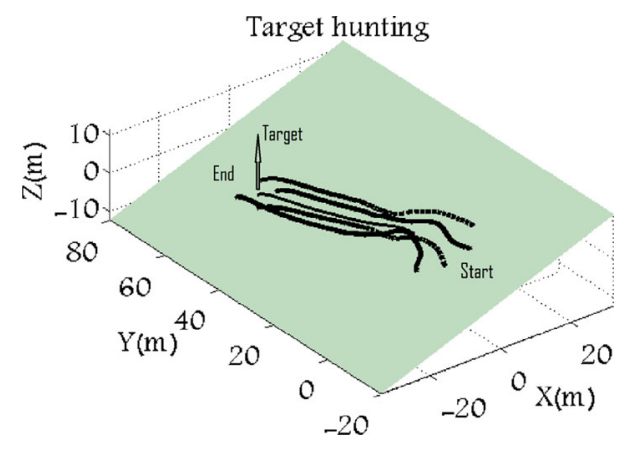

Fig. 9 Following and hunting the target with four robots on ramp surface

Figure 10 shows initial and final place of the (Fig. 11) target using Black spot.

Figure 12 Showing Relative distance on ramp surface between robots and the mobile target. As it is seen in Fig. 12 the distance between robots and the target is converged by an equilibrium distance: $\mathrm{Rt}=5(\mathrm{~m})$ and also it is see that the distance between robots is in a manner that no accident occurs. Relative distance related to two other surfaces is shown in Appendix 6.

Figure 13 showing robots velocity and the target in ramp surface. Reduction in velocity of robots and the target means gradual hint of the target. As it is seen the target increases 


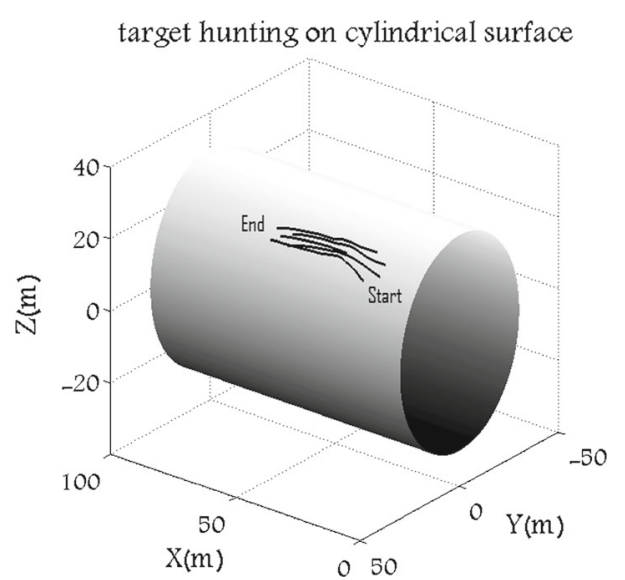

Fig. 10 Following and hunting the target with four robots on cylindrical surface

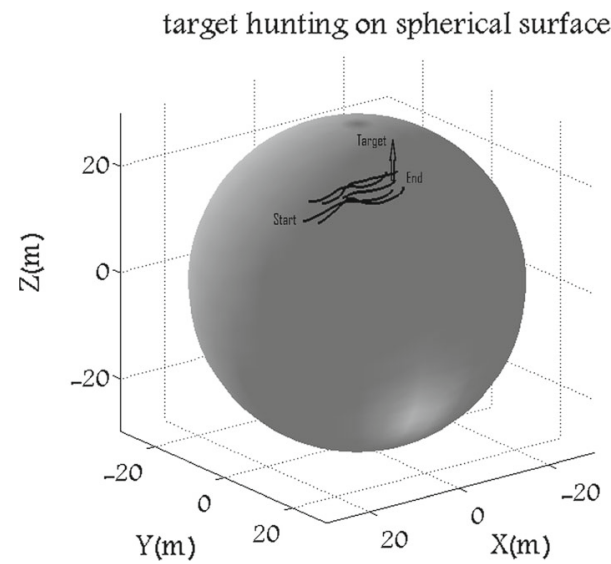

Fig. 11 Following and hunting the target with four robots on spherical surface
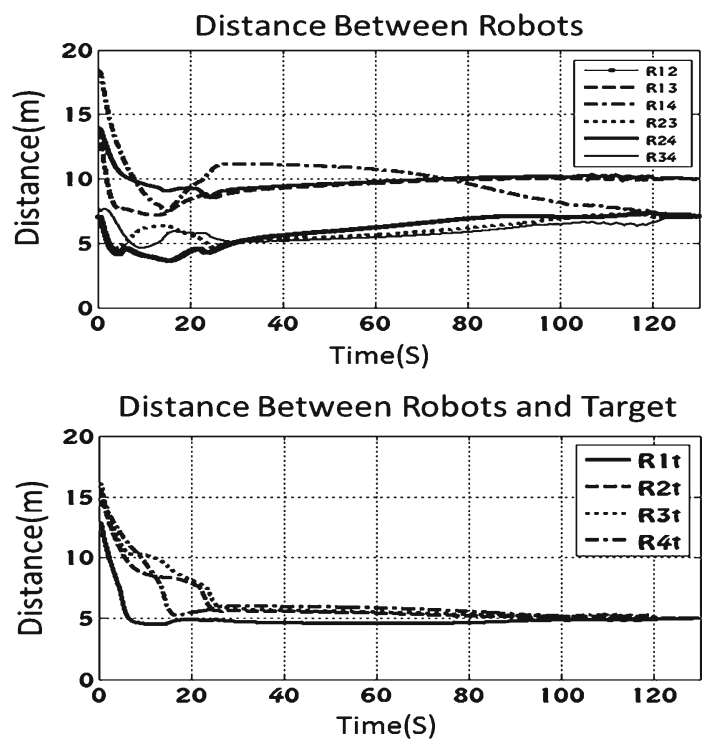

Fig. 12 Distances between the target and robots (down), and distances between robots (up) on ramp surface
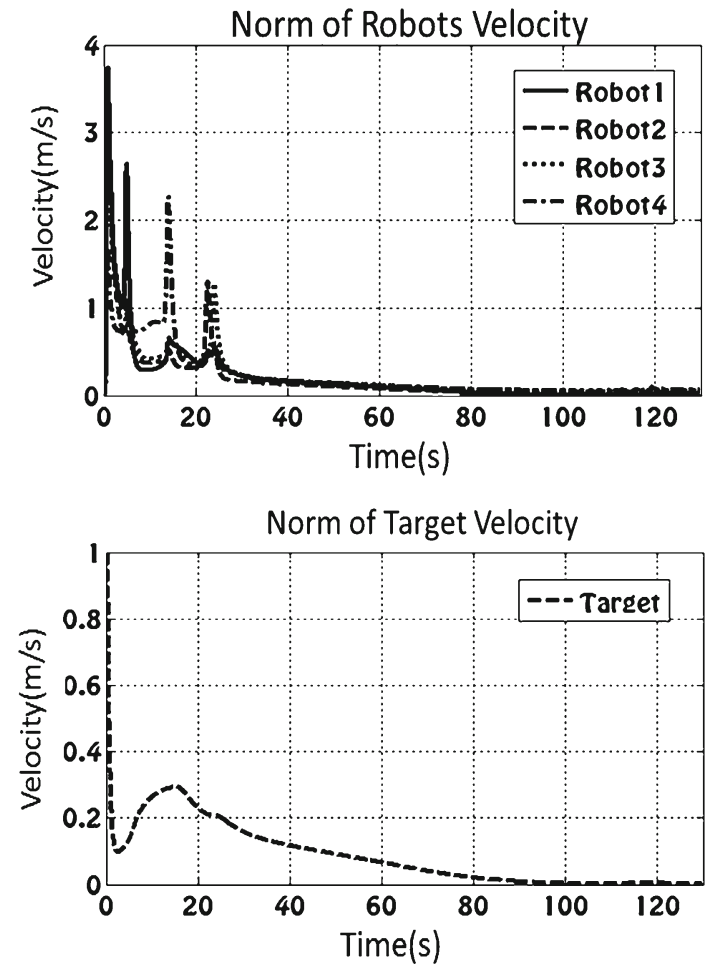

Fig. 13 Velocity of robots center (up), and velocity of target (down) on ramp surface

because robots come close to it but reduces as circulation occurs. The velocity of robot and the target related to two other surfaces are shown in Appendix 6.

Figure 14 shows control torques over the steering and front wheel by considering the effect of filtering and saturation of actuators in ramp surface. As it is seen entered torque over front wheel at final is slightly different with zero and it is because the robot is in ramp surface and for being stable on ramp surface must enter a little torque over front wheel till the effect of weigh power inhibited. Torque diagram shows rational results mechanically. The robot torques of the two other surfaces is shown in Appendix 6.

\subsection{Showing estimation variables and the error}

In this section we show estimated variables and show estimation error. State variables are estimated by extended Kalman filter and smoother.

Figure 15 Showing the estimation of relative distance between two robots.

As we will see in Fig. 15 the relative distance using extended Kalman filter is estimated precisely and the estimation error is low and acceptable.

Figure 16 shows this procedure. By comparing two diagrams we see that extended Kalman smoother gets a better estimation and has less chaos. 

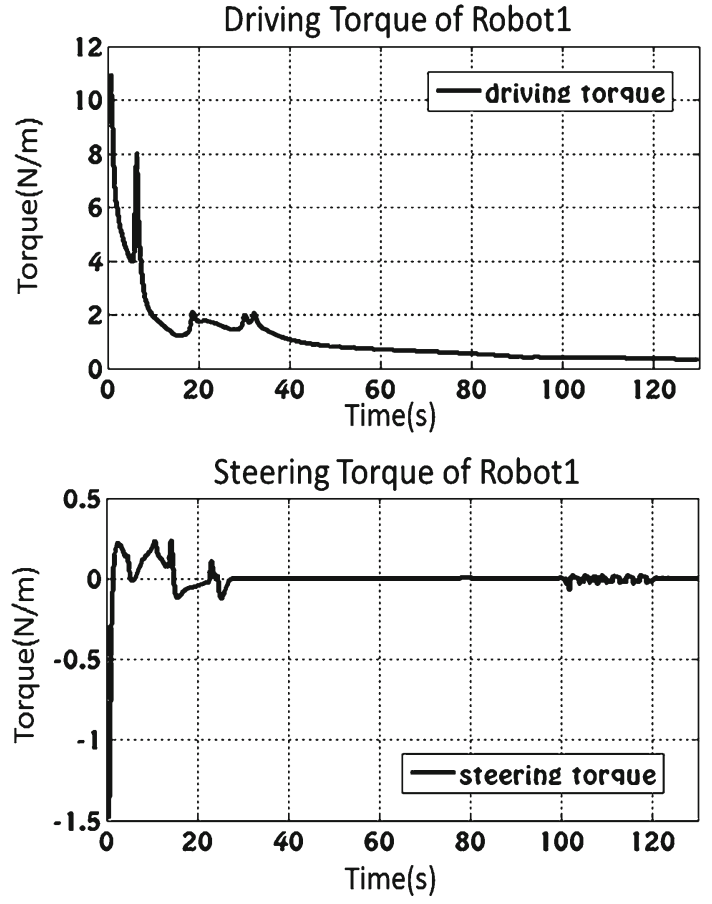

Fig. 14 Driving torque (down), and steering torque (up) on ramp surface

Figures 17 and 18 showing relative distance estimation between robot and the target.

Like estimation of relative distance between two robots, the relative distance between (Figs. 19, 20) robot and the target is estimated by less error and also using smoother we have a better estimation.

\subsection{Following missions around the target}

As, it was mentioned based (Fig. 21) on the type of the mission the relative distance between robots is adjusted. For the hunt, relative distance between the robots, according to the distance to the target $R_{t}=5(\mathrm{~m})$, is selected and it is given based on the geometry of four robots and the distance between the robots when the robot hunting $R_{R}=5 \sqrt{2}(\mathrm{~m})$ is obtained and For the follow, relative distance between the robots Reduced $R_{R}=0.7 \times 5 \sqrt{2}(\mathrm{~m})$.

In Fig. 22, body angle and steering angle are shown in robot1. As it is evident the robot body angle goes toward desired acceleration angle and moves toward the target from its front.

\subsection{Following and hunting the target with five and three robots}

In Figs. 23 and 24 we show that group algorithm is scalable.
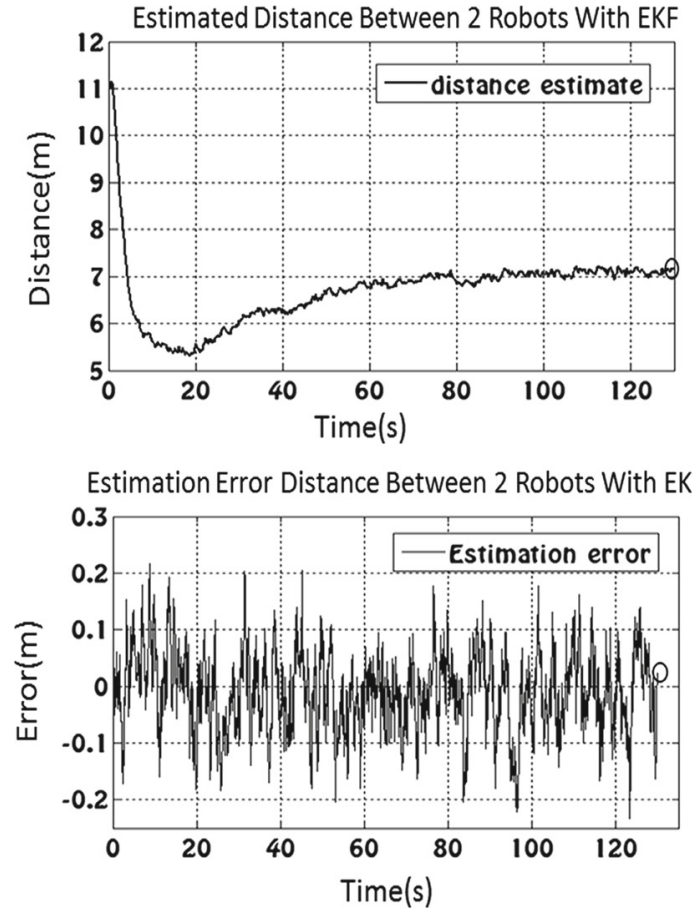

Fig. 15 Estimated relative distance between two robots and its estimation error with EKF
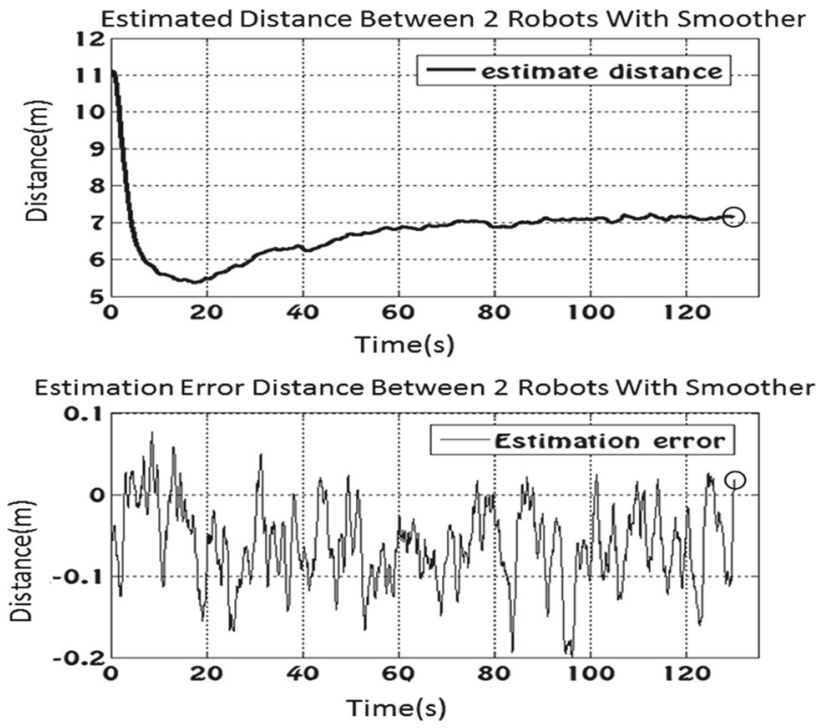

Fig. 16 Estimated relative distance between two robots and its estimation error with ERTS

\section{Conclusion}

In this paper, the hunt problem and the target chase with holonomic constrain by wheeler robots, with non-holonomic constrain has been studied. It is supposed that implemented measurement by chasing robots have been incomplete and is by noise. In fact, state variables are unknown and estimator is used. Mobile target dynamic is considered in a manner that is escaping continuously by robots. Based on simulation 


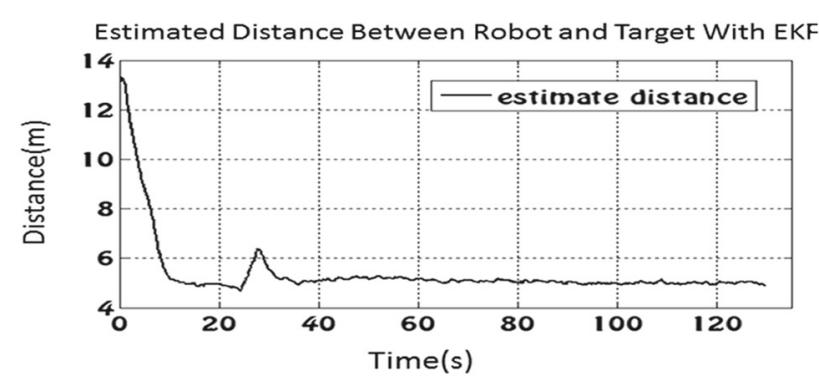

Estimation Error Distance Between 2 Robots With EKF

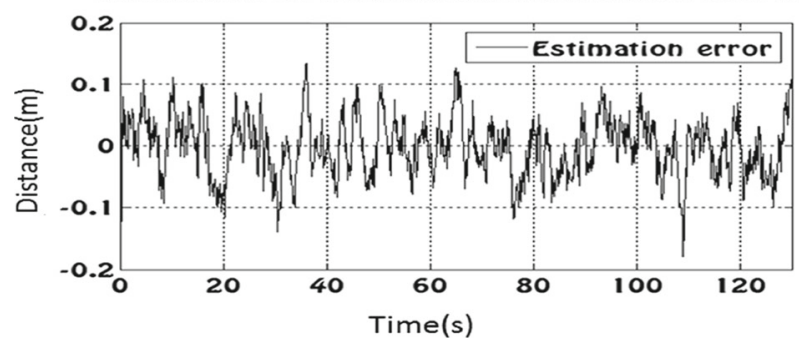

Fig. 17 Estimated relative distance between robot and target and its estimation error with EKF

Estimated Distance Between Robot and Target With Smoother

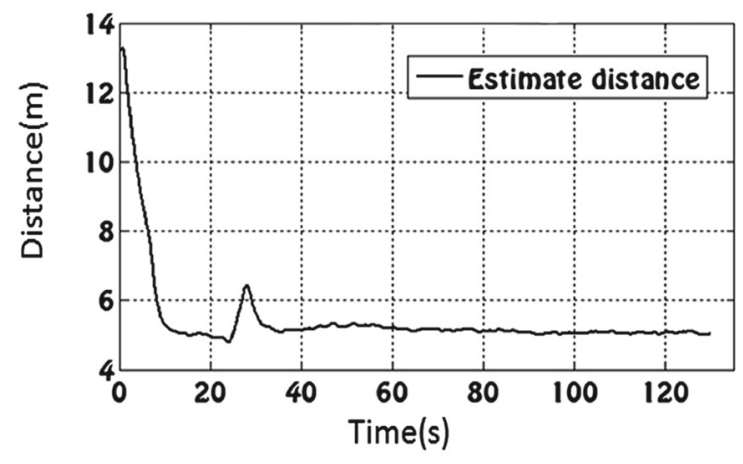

Estimation Error Distance Between 2 Robots With Smoother

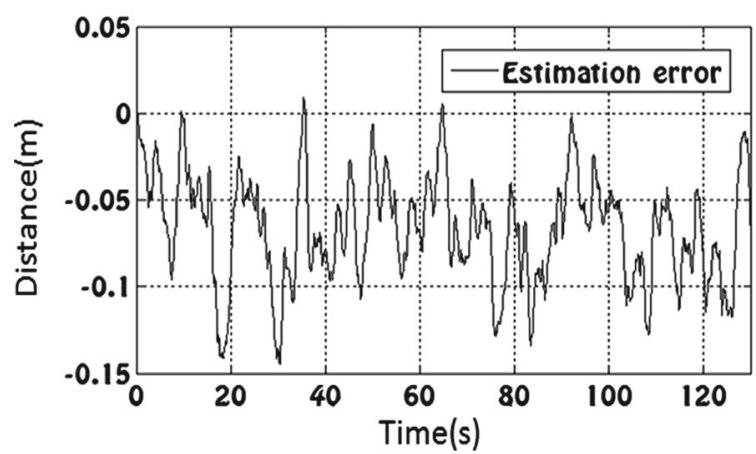

Fig. 18 Estimated relative distance between robot and target and its estimation error with ERTS

results, it is seen that control algorithm is suitable and there is no need to use complicated algorithms.

One of the reasons for the correct implementation of the operation is correct analyzes of inertial mass and using variable control coefficients. Meanwhile, based on results

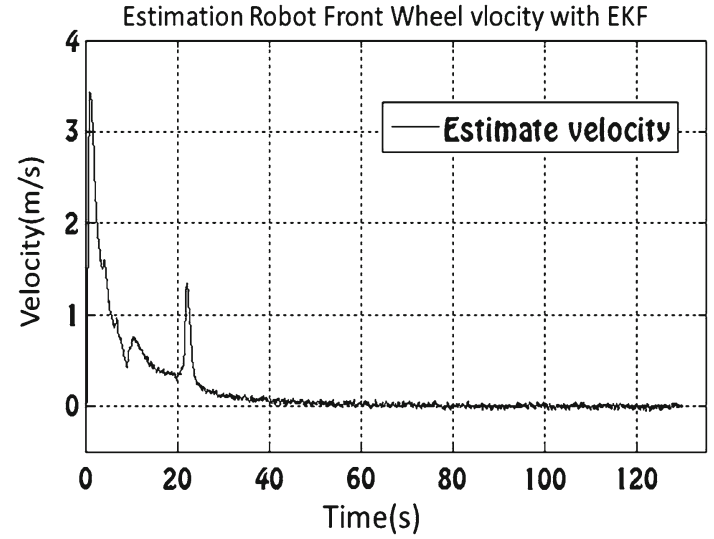

Fig. 19 Estimated front wheel velocity of robot with EKF

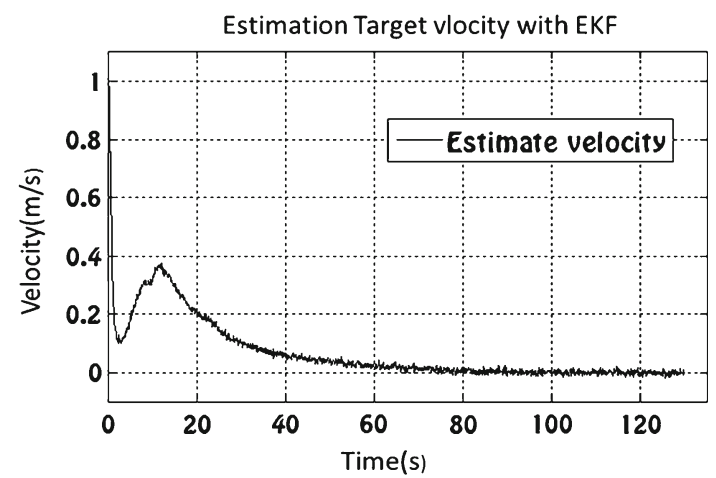

Fig. 20 Estimated norm of target velocity with EKF

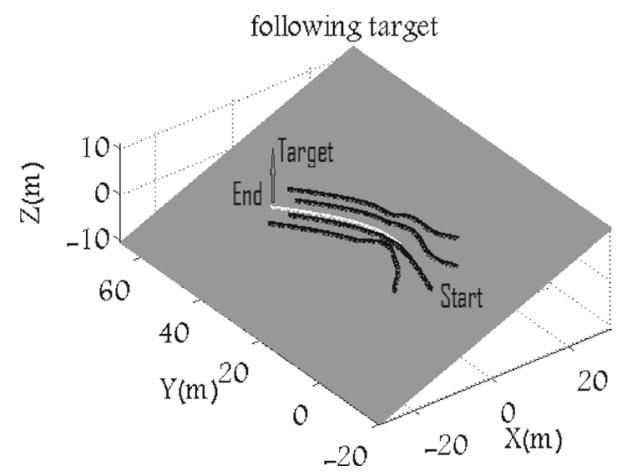

Fig. 21 How the target is followed and how coverage is made around it

it is seen that the operation has been implemented precisely. Using group operation with estimation algorithms is successful. Output of controllers is torque that we can analyze the possibility of the maneuver.

Group algorithm is in a manner that robots move in desired direction continuously and as we see the movement is continuously toward front. The operation is implemented in a manner that by changing control gains both operations of hunt and covering are done correctly and the operation is correct in three surfaces. Based on these results, we can 

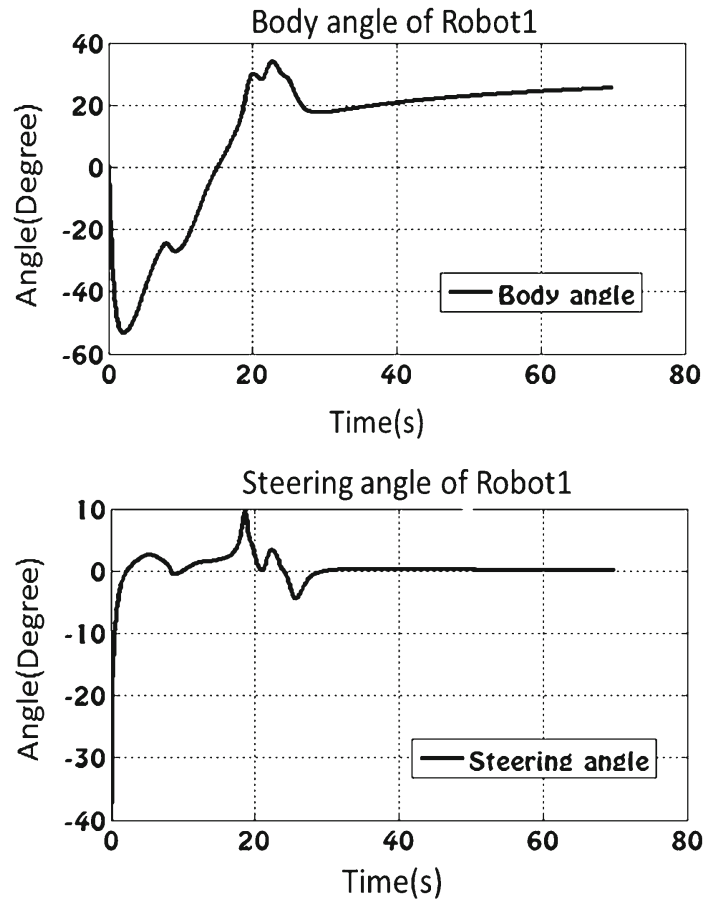

Fig. 22 Steering angle (down) and body angle of robot 1 (up)

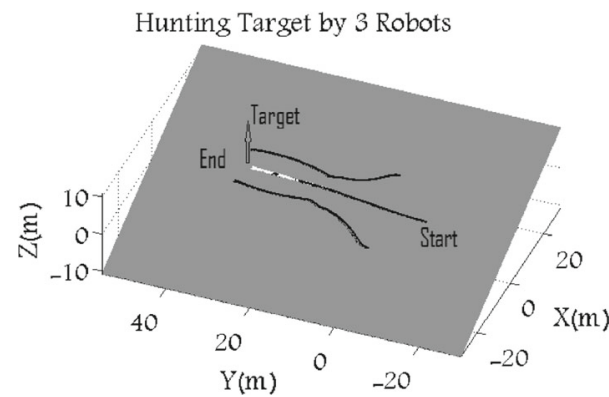

Fig. 23 Following and hunting the target with three robots on ramp surface

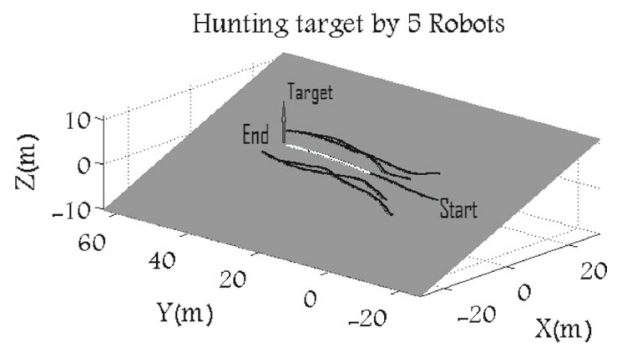

Fig. 24 Following and hunting the target with five robots on ramp surface

consider it a security fence around a leader. This is a pace for doing real group operation. Future work in this field is mostly about improving indirect control of body angles, estimating environmental disturbances and using them in the control procedure, controlling car-like robots equipped with special accessories, preventing robots from rolling while per- forming group maneuvers, estimating surrounding obstacles, performing different kinds of maneuver while obstacles are present and performing practical operations; for example, in traffic engineering.

\section{Appendices}

Appendix 1: Variable coefficients

$$
\begin{aligned}
& a_{6}=\frac{-S \beta}{r}+\frac{b}{r L} C \beta, a_{7}=\frac{-S \beta}{r}-\frac{b}{r L} C \beta, a_{8}=\frac{-C \beta}{L} \\
& a_{9}=m_{b}+2 m_{w} L^{2} a_{3}^{2}-m_{b} L(L-a) a_{3}^{2}+2 m_{w} b^{2} a_{3}^{2} \\
& +\left(I_{b z}+2 I_{w z}\right) a_{3}^{2}+I_{w y}\left(a_{1}^{2}+a_{2}^{2}\right) \\
& a_{10}=m_{b} a_{3} a_{8} L(L-a)-2 m_{w}\left(L^{2}-b^{2}\right) a_{3} a_{8} \\
& -I_{b z} a_{3} a_{8}-2 I_{w z} a_{3} a_{8}+I_{w y}\left(a_{1} a_{6}+a_{2} a_{7}\right) \\
& a_{11}=-m_{b} a_{3}^{2} a_{8} L(L-a)-\left(2 m_{w}+m_{b}\right) L^{2}\left(a_{8} a_{3}^{2}+a_{3} a_{8}^{2}\right) \\
& a_{12}=-2 m_{w} g S \alpha C \theta C \beta-m_{b} g S \alpha C(\theta+\beta) \\
& a_{13}=S \beta C \theta\left(-\frac{a}{L} a_{3} u_{1}-u_{2}\right)+C \beta S \theta\left(-\frac{a}{L} u_{2}-a_{3} u_{1}\right) \\
& a_{14}=S \beta S \theta\left(-\frac{a}{L} a_{3} u_{1}-u_{2}\right)+C \beta C \theta\left(\frac{a}{L} u_{2}+a_{3} u_{1}\right) \\
& a_{15}=\frac{1}{R_{C}+r}\left(S \beta S \theta-\frac{a}{L} C \beta C \theta\right) \text {, } \\
& a_{16}=\left(S \beta C \theta+\frac{a}{L} C \beta S \theta\right) \\
& a_{17}=m_{b}\left(\left(L a_{8}\right)^{2}+\left(a a_{3}\right)^{2}\right)+m_{w} r^{2}\left(a_{4}^{2}+a_{5}^{2}\right) \\
& +\left(\frac{a_{2}}{R_{C}+r}\right)^{2}(C \theta)^{2} I_{b x}+\left(\frac{a_{2}}{R_{C}+r}\right)^{2}(S \theta)^{2} I_{b \phi} \\
& +a_{3}^{2} I_{b r}+2\left(\frac{a_{2}}{R_{C}+r}\right)^{2}(C \theta)^{2} I_{w x} \\
& +2\left(\frac{a_{2}}{R_{C}+r}\right)^{2}(S \theta)^{2} I_{w \phi} \\
& +2 I_{w \phi} \frac{a_{2} a_{4}}{R_{C}+r} S \theta+2 I_{w \phi} \\
& \frac{a_{2} a_{5}}{R_{C}+r} S \theta+\left(a_{4}^{2}+a_{5}^{2}\right) I_{w \phi}+2 a_{3}^{2} I_{w r} \\
& a_{18}=-m_{b} a_{8} a_{3}\left(L^{2}-a^{2}\right)+m_{w} r^{2}\left(a_{4} a_{6}+a_{5} a_{7}\right) \\
& +I_{w \phi} a_{5} a_{7}-\frac{a_{2}}{R_{C}+r} a_{15}(C \theta)^{2} I_{b x} \\
& -\frac{a_{2}}{R_{C}+r} a_{15}(S \theta)^{2} I_{b \phi}-I_{w \phi} a_{5} a_{15} S \theta \\
& -2 \frac{a_{2}}{R_{C}+r} a_{15}(C \theta)^{2} I_{w x}-2 \frac{a_{2}}{R_{C}+r} a_{15} \\
& (C \theta)^{2} I_{w x}-2 \frac{a_{2}}{R_{C}+r} a_{15}(S \theta)^{2} I_{w \phi} \\
& +I_{w \phi} \frac{a_{2} a_{7}}{R_{C}+r} S \theta+I_{w \phi} \frac{a_{2} a_{6}}{R_{C}+r} S \theta \\
& -I_{w \phi} a_{4} a_{15} S \theta+I_{w \phi} a_{4} a_{6}+2 I_{w r} a_{3} a_{8}
\end{aligned}
$$




$$
\begin{aligned}
& a_{19}=\frac{a_{1} a_{2} a_{3}}{R_{C}+r}\left((C \theta)^{2} I_{b x}+(S \theta)^{2} I_{b \phi}\right) \\
& +\left(\frac{a_{2}}{R_{C}+r}\right)^{2} a_{3} \operatorname{C\theta S} \theta\left(I_{b \phi}-I_{b x}\right) \\
& +2 I_{w x} \frac{a_{1} a_{2} a_{3}}{R_{C}+r} C \theta+I_{w \phi} \frac{a_{1} a_{2} a_{3}}{R_{C}+r} C \theta \\
& +2\left(\frac{a_{2}}{R_{C}+r}\right)^{2} a_{3} S \theta C \theta \\
& \left(I_{w \phi}+I_{w x}\right)+2 I_{w \phi} \frac{a_{1} a_{2} a_{3}}{R_{C}+r}(S \theta)^{2} \\
& +I_{w \phi} a_{1} a_{4} a_{3} S \theta+\frac{a_{4} a_{2} a_{3}}{R_{C}+r} C \theta\left(I_{w r}-I_{w x}\right) \\
& +I_{w \phi} \frac{a_{5} a_{2} a_{3}}{R_{C}+r} C \theta+I_{w \phi} a_{5} a_{1} a_{3} S \theta \\
& +\frac{a_{5} a_{2} a_{3}}{R_{C}+r} C \theta\left(I_{w r}-I_{w x}\right) \\
& a_{20}=m_{b} g S \phi S \theta\left(a a_{3} C \theta-L a_{8} S \theta\right)-m_{w} g r S \phi S \theta\left(a_{4}+a_{5}\right) \\
& a_{21}=\left(m_{b} a^{2}+2 m_{w} b^{2}+\frac{a^{2} I_{b \gamma^{\prime}}}{\left(R_{s}+r\right)^{2}}+\frac{2 a^{2} I_{w \gamma^{\prime}}}{\left(R_{s}+r\right)^{2}}\right. \\
& \left.+2 I_{w r-} \frac{2 b^{2} I_{w \delta^{\prime}}}{r^{2}}\right) a_{3}^{2} \\
& +\left(m_{b}+\frac{I_{b \delta^{\prime}}}{\left(R_{S}+r\right)^{2}}-\frac{2\left(R_{S}+2 r\right) I_{w \delta^{\prime}}}{\left(R_{S}+r\right) r}\right) L^{2} a_{8}^{2} \\
& +\left(-2 m_{w}+\frac{2\left(R_{s}+2 r\right) I_{w r}}{\left(R_{s}+r\right) L}\right) L^{2} a_{8} a_{3} \\
& a_{22}=-2 m_{w} L^{2} a_{3}^{2}-\frac{2 L\left(R_{S}+2 r\right) I_{w r}}{\left(R_{S}+r\right)} a_{8}^{2}+\left(m_{b}\left(L^{2}-a^{2}\right)\right. \\
& -2 m_{w} b^{2}-\frac{a^{2} I_{b \gamma^{\prime}}}{\left(R_{s}+r\right)^{2}}-I_{b r}-\frac{2 a^{2} I_{w \gamma^{\prime}}}{\left(R_{s}+r\right)^{2}}-2 I_{w r} \\
& \left.-\frac{2 b^{2} I_{w \delta^{\prime}}}{r^{2}}-\frac{2 L^{2}\left(R_{s}+2 r\right) I_{w \delta^{\prime}}}{\left(R_{s}+r\right) r}\right) a_{3} a_{8} \\
& a_{23}=\left(-\frac{a L I_{b r}}{\left(R_{S}+r\right)^{2}}+\frac{2 a L I_{w r}}{\left(R_{s}+r\right)^{2}}-\frac{2 L^{2} I_{w r}}{r\left(R_{s}+r\right)}\right. \\
& \left.-\frac{2 L^{2} I_{w r}}{\left(R_{S}+r\right)^{2}}-\frac{L^{2} I_{b r}}{\left(R_{S}+r\right)^{2}}\right) a_{3}^{2} a_{8} \\
& \left.-\frac{2 L^{2}\left(R_{s}+2 r\right) a I_{w \delta^{\prime}}}{\left(R_{S}+r\right)^{3}}\right) a_{3} a_{8}^{2} \\
& a_{24}=-2 m_{w} g S \beta C \delta S \theta-m_{b} g S \delta a_{1}
\end{aligned}
$$

Appendix 2: Velocity and angular velocity

\section{Cylindrical surface}

$$
\begin{aligned}
v_{A} & =u_{1} e_{x^{\prime}}, v_{L}=v_{A}-b \dot{\theta} e_{x}-L \dot{\theta} e_{\phi}=\dot{\phi}_{L} r e_{x} \\
v_{R} & =v_{A}+b \dot{\theta} e_{x}-L \dot{\theta} e_{\phi}=\dot{\phi}_{R} r e_{x}, v_{b}=v_{A}-(L-a) \dot{\theta} e_{\phi} \\
& =\left(R_{c}+r\right) \dot{\phi} e_{\phi}+\dot{X} e_{x} \\
\omega_{b} & =-\dot{\phi} e_{X}+\dot{\theta} e_{r}, \omega_{R}=\omega_{b}+\dot{\phi}_{R} e_{\varphi}, \omega_{L}=\omega_{b}+\dot{\phi}_{L} e_{\varphi}
\end{aligned}
$$

Spherical surface

$$
\begin{aligned}
v_{A}= & u_{1} e_{\gamma^{\prime \prime}}, v_{L}=v_{A}-b(\dot{\gamma} C \delta+\dot{\theta}) e_{\gamma^{\prime}}+L(\dot{\gamma} C \delta+\dot{\theta}) e_{\delta^{\prime}} \\
= & \dot{\phi}_{L} r e_{\gamma^{\prime}}, v_{R}=v_{A}+b(\dot{\gamma} C \delta+\dot{\theta}) e_{\gamma^{\prime}} \\
& +L(\dot{\gamma} C \delta+\dot{\theta}) e_{\delta^{\prime}}=\dot{\phi}_{R} r e_{\gamma^{\prime}} \\
v_{b}= & v_{A}+(L-a)(\dot{\gamma} C \delta+\dot{\theta}) e_{\delta^{\prime}}=\left(R_{S}+r\right) \dot{\delta} e_{\delta} \\
& +\left(R_{S}+r\right) \dot{\gamma} S \delta e_{\gamma} \\
\omega_{b}= & \dot{\delta} C \gamma e_{X}+\dot{\gamma} e_{Y}-\dot{\delta} S \gamma e_{Z}+\dot{\theta} e_{r}, \\
\omega_{R}= & \omega_{b}-\dot{\phi}_{R} e_{\delta^{\prime}}, \omega_{L}=\omega_{b}-\dot{\phi}_{L} e_{\delta^{\prime}}
\end{aligned}
$$

Appendix 3: Generalized coordinates in each surfaces

Ramp surface

$\left[\begin{array}{c}\ddot{q}_{E 1} \\ \ddot{q}_{E 2}\end{array}\right]=\left[\begin{array}{c}\ddot{X}^{\prime} \\ \ddot{Y}^{\prime}\end{array}\right],\left[\begin{array}{c}\dot{q}_{E 1} \\ \dot{q}_{E 2}\end{array}\right]=\left[\begin{array}{c}\dot{X}^{\prime} \\ \dot{Y}^{\prime}\end{array}\right],\left[\begin{array}{c}q_{E 1} \\ q_{E 2}\end{array}\right]=\left[\begin{array}{c}X^{\prime} \\ Y^{\prime}\end{array}\right]$

Cylindrical surface

$$
\begin{aligned}
& {\left[\begin{array}{l}
\ddot{q}_{E 1} \\
\ddot{q}_{E 2}
\end{array}\right]=\left[\begin{array}{l}
\ddot{X} \\
\left(R_{c}+r\right) \ddot{\phi}
\end{array}\right],\left[\begin{array}{l}
\dot{q}_{E 1} \\
\dot{q}_{E 2}
\end{array}\right]=\left[\begin{array}{l}
\dot{X} \\
\left(R_{c}+r\right) \dot{\phi}
\end{array}\right],} \\
& {\left[\begin{array}{l}
q_{E 1} \\
q_{E 2}
\end{array}\right]=\left[\begin{array}{l}
X \\
\left(R_{c}+r\right) \phi
\end{array}\right]}
\end{aligned}
$$

Spherical surface

$$
\begin{aligned}
& {\left[\begin{array}{l}
\ddot{q}_{E 1} \\
\ddot{q}_{E 2}
\end{array}\right]=\left[\begin{array}{l}
\left(R_{S}+r\right) \ddot{\delta} \\
\left(R_{S}+r\right) S \delta \ddot{\gamma}
\end{array}\right],\left[\begin{array}{l}
\dot{q}_{E 1} \\
\dot{q}_{E 2}
\end{array}\right]=\left[\begin{array}{l}
\left(R_{S}+r\right) \dot{\delta} \\
\left(R_{S}+r\right) S \delta \dot{\gamma}
\end{array}\right],} \\
& {\left[\begin{array}{l}
q_{E 1} \\
q_{E 2}
\end{array}\right]=\left[\begin{array}{l}
\left(R_{S}+r\right) \delta \\
\left(R_{S}+r\right) S \delta \gamma
\end{array}\right]}
\end{aligned}
$$

Appendix 4: Relative equation of motion between two robots

\section{Ramp surface}

$$
\left[\begin{array}{l}
\dot{u}_{1}^{i} \\
\dot{u}_{2}^{i} \\
\dot{\theta}_{i} \\
\dot{\rho}_{i j} \\
\dot{\vartheta}_{i j}
\end{array}\right]=\left[\begin{array}{l}
\frac{M_{i}}{R a_{9}^{i}}-\frac{a_{11}^{i}}{a_{9}^{i}}\left(u_{1}^{i}\right)^{2}-\frac{a_{10}^{i}}{a_{9}^{i}} u_{1}^{i} u_{2}^{i}+\frac{a_{12}^{i}}{a_{9}^{i}} \\
\frac{T_{i}}{C_{b}} \\
a_{3}^{i} u_{1}^{i} \\
u_{1}^{i} S \alpha_{i j}-u_{1}^{j} S \vartheta_{i j} \\
\frac{u_{1}^{i} C \alpha_{i j}-u_{1}^{j} C \vartheta_{i j}}{\rho_{t i}}-\dot{\theta}_{i}
\end{array}\right]
$$

Cylindrical surface

$$
\left[\begin{array}{l}
\dot{u}_{1}^{i} \\
\dot{u}_{2}^{i} \\
\dot{\theta}_{i} \\
\dot{\rho}_{i j} \\
\dot{\vartheta}_{i j}
\end{array}\right]=\left[\begin{array}{l}
\frac{M_{i}}{R a_{17}^{i}}-\frac{a_{19}^{i}}{a_{17}^{i}}\left(u_{1}^{i}\right)^{2}-\frac{a_{18}^{i}}{a_{17}^{i}} u_{1}^{i} u_{2}^{i}+\frac{a_{20}^{i}}{a_{17}^{i}} \\
\frac{T_{i}}{C_{b}} \\
a_{3}^{i} u_{1}^{i} \\
u_{1}^{i} S \alpha_{i j}-u_{1}^{j} S \vartheta_{i j} \\
\frac{u_{1}^{i} C \alpha_{i j}-u_{1}^{j} C \vartheta_{i j}}{\rho_{t i}}-\dot{\theta}_{i}
\end{array}\right]
$$


Spherical surface

$\left[\begin{array}{l}\dot{u}_{1}^{i} \\ \dot{u}_{2}^{i} \\ \dot{\theta}_{i} \\ \dot{\rho}_{i j} \\ \dot{\vartheta}_{i j}\end{array}\right]=\left[\begin{array}{l}\frac{M_{i}}{R a_{21}^{i}}-\frac{a_{23}^{i}}{a_{21}^{i}}\left(u_{1}^{i}\right)^{2}-\frac{a_{22}^{i}}{a_{21}^{i}} u_{1}^{i} u_{2}^{i}+\frac{a_{24}^{i}}{a_{21}^{i}} \\ \frac{T_{i}}{C_{b}} \\ a_{3}^{i} u_{1}^{i} \\ u_{1}^{i} S \alpha_{i j}-u_{1}^{j} S \vartheta_{i j} \\ \frac{u_{1}^{i} C \alpha_{i j}-u_{1}^{j} C \vartheta_{i j}}{\rho_{t i}}-\dot{\theta}_{i}\end{array}\right]$

Appendix 5: Relative equation of motion between robot and target

Ramp surface

$\left[\begin{array}{l}\ddot{X}_{t}^{\prime} \\ \ddot{Y}_{t}^{\prime} \\ \dot{\rho}_{t i} \\ \dot{\vartheta}_{t i}\end{array}\right]=\left[\begin{array}{l}\frac{K_{t}}{m_{t}} F_{X^{\prime}}^{t}-\frac{C_{t}}{m_{t}} \dot{X}_{t}^{\prime} \\ \frac{K_{t}}{m_{t}} F_{Y^{\prime}}^{t}-\frac{C_{t}}{m_{t}} \dot{Y}_{t}^{\prime} \\ v_{t} S \alpha_{t i}-v_{i} S \vartheta_{t i} \\ \frac{v_{t} C \alpha_{t i}-v_{i} C \vartheta_{t i}}{\rho_{t i}}-\dot{\theta}_{i}\end{array}\right], B^{t}=\left[\begin{array}{llll}\frac{K_{t}}{m_{t}} & 0 & 0 & 0 \\ 0 & \frac{K_{t}}{m_{t}} & 0 & 0\end{array}\right]^{T}$

Cylindrical surface

$$
\begin{aligned}
& {\left[\begin{array}{l}
\ddot{X}_{t} \\
\ddot{\phi}_{t} \\
\dot{\rho}_{t i} \\
\dot{\vartheta}_{t i}
\end{array}\right]=\left[\begin{array}{l}
\frac{K_{t}}{m_{t}} F_{X}^{t}-\frac{C_{t}}{m_{t}} \dot{X}_{t} \\
\frac{K_{t}}{m_{t}\left(R_{c}+r\right)} F_{\phi}^{t}-\frac{C_{t}}{m_{t}} \dot{\phi}_{t} \\
v_{t} S \alpha_{t i}-v_{i} S \vartheta_{t i} \\
\frac{v_{t} C \alpha_{t i}-v_{i} C \vartheta_{t i}}{\rho_{t i}}-\dot{\theta}_{i}
\end{array}\right],}
\end{aligned}
$$

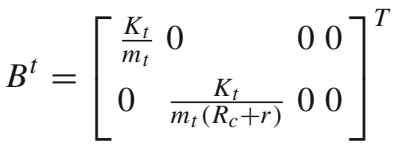

Spherical surface

$$
\begin{aligned}
& {\left[\begin{array}{l}
\ddot{\delta}_{t} \\
\ddot{\gamma}_{t} \\
\dot{\rho}_{t i} \\
\dot{\vartheta}_{t i}
\end{array}\right]=\left[\begin{array}{l}
\frac{K_{t}}{m_{t\left(R_{s}+r\right)}} F_{\delta}^{t}-\frac{C_{t}}{m_{t}} \dot{\delta}_{t} \\
\frac{K_{t}}{m_{t}\left(R_{s}+r\right) S \delta_{t}} F_{\gamma}^{t}-\frac{C_{t}}{m_{t}} \dot{\gamma}_{t} \\
v_{t} S \alpha_{t i}-v_{i} S \vartheta_{t i} \\
\frac{v_{t} C \alpha_{t i}-v_{i} C \vartheta_{t i}}{\rho_{t i}}-\dot{\theta}_{i}
\end{array}\right],} \\
& B^{t}=\left[\begin{array}{llll}
\frac{K_{t}}{m_{t}} & 0 & 0 & 0 \\
0 & \frac{K_{t}}{m_{t}\left(R_{s}+r\right) S \delta_{t}} & 0 & 0
\end{array}\right]^{T}
\end{aligned}
$$

Appendix 6: Diagram

Relative distance on cylindrical surface
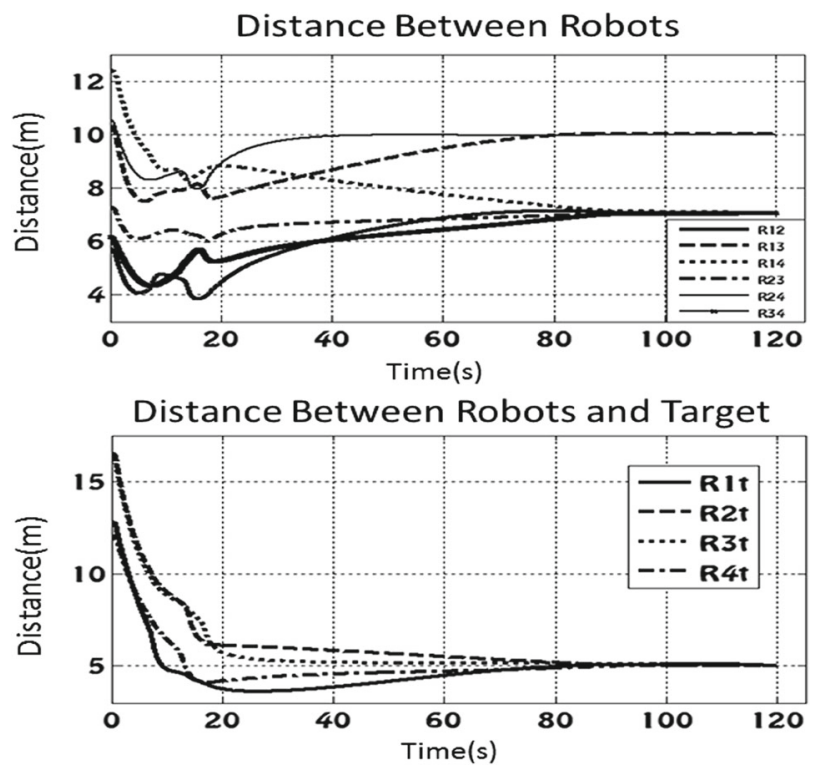

Relative distance on spherical surface
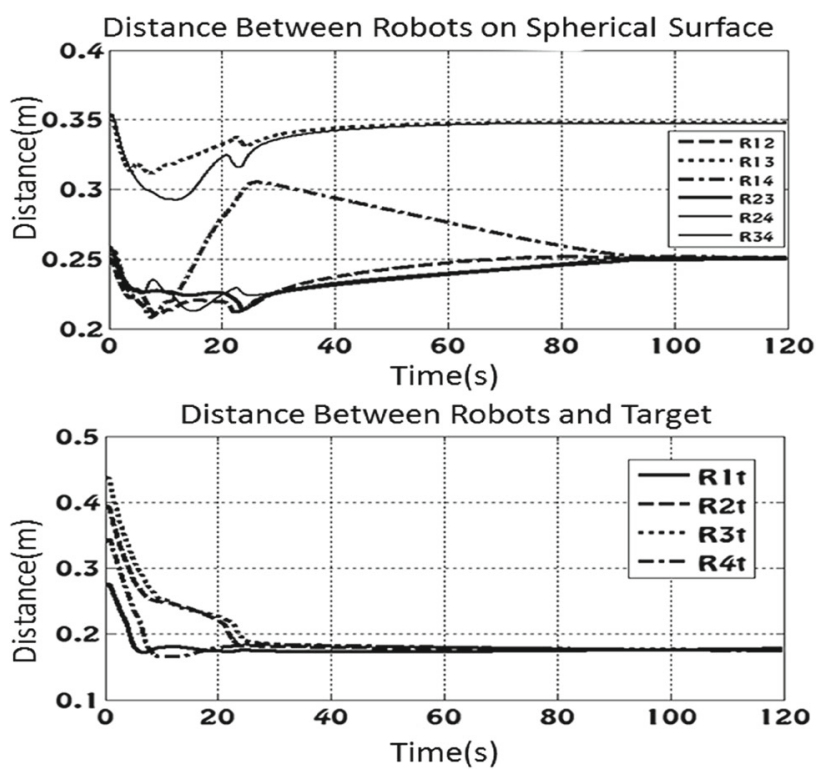
Robot and target velocity

Cylindrical surface
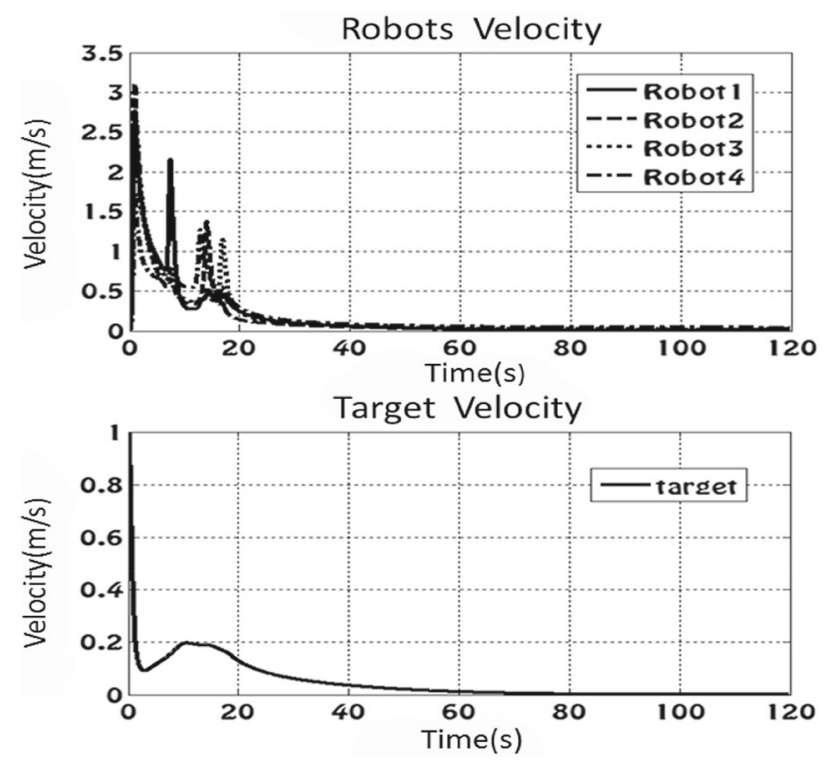

Spherical surface
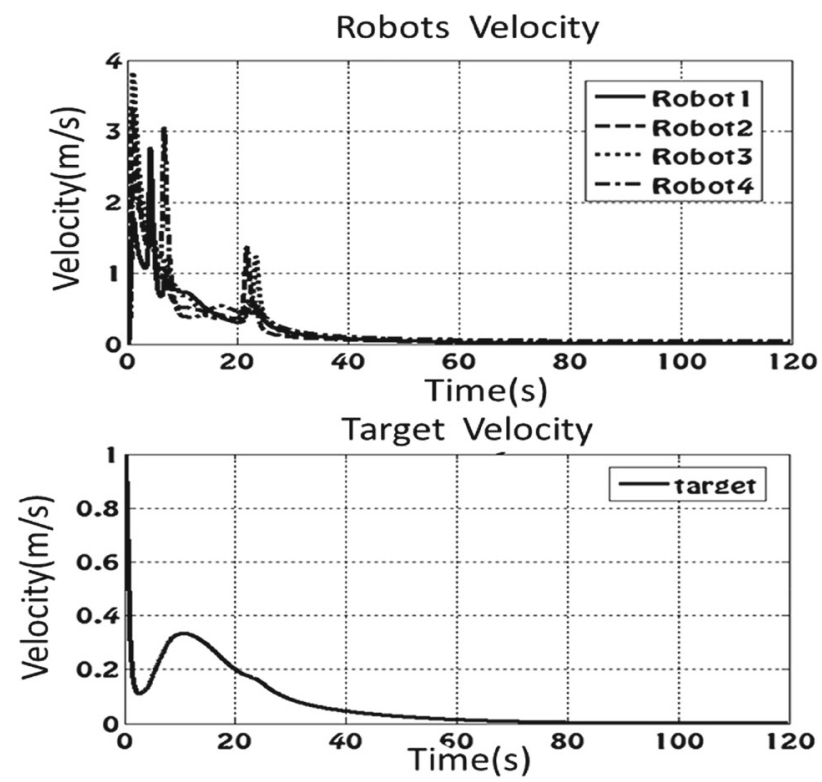

Control torque

Cylindrical surface
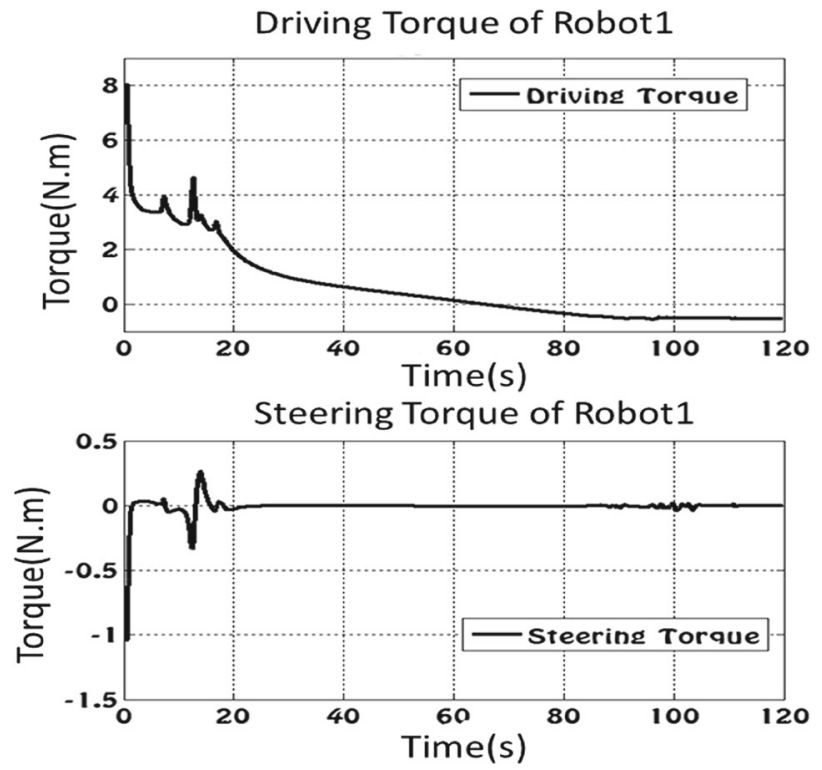

Spherical surface
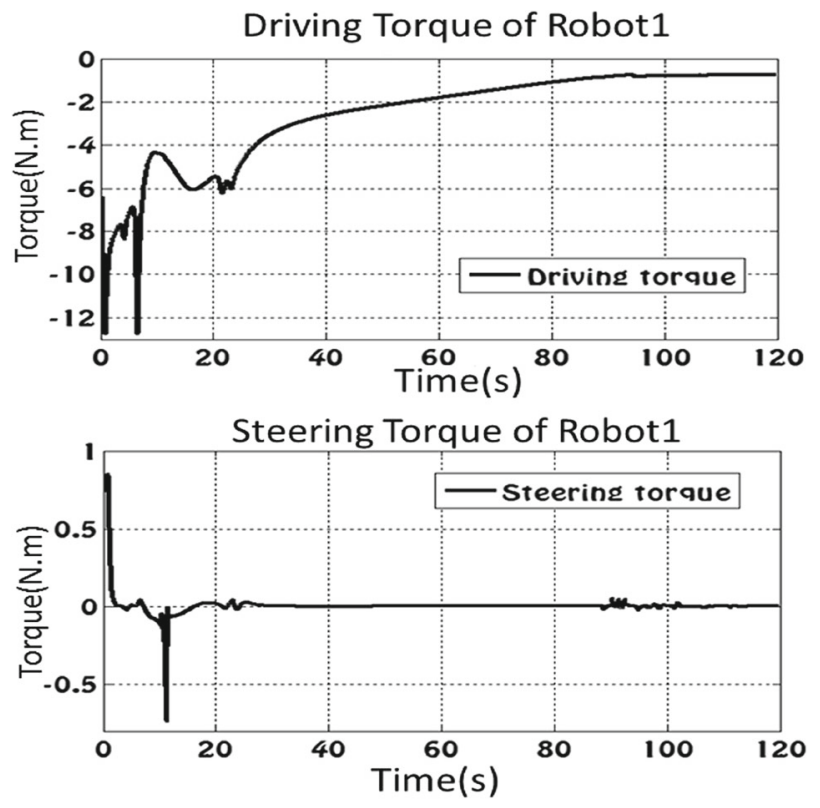


\section{References}

1. Jun $O$ (2006) Multi-agent robot systems as distributed autonomous systems. Adv Eng Inf 20(1):59-70

2. Sayyaadi H, Moarref M (2011) A distributed algorithm for proportional task allocation in networks of mobile agents. IEEE J Autom Control 56:405-410

3. Sayyaadi H, Dostmohammadian MR (2011) Finite time consensus in directed switching network topologies and time-delayed communications. Sci Iran 18(1):77-85

4. Wang YT, Chen YC, Lin MC (2009) Dynamic object tracking control for a non-holonomic wheeled autonomous robot. Tamkang $\mathrm{J}$ Sci Eng 12(3):339-350

5. Matveev AS, Teimooria H, Savkin AV (2011) Range-only measurements based target following for wheeled mobile robots. Automatica 47(1):177-184

6. Agustinus T, Hong B (2009) A path following control of an unmanned autonomous forklift. Int J Control Autom Syst 7(1):113122

7. Bemporad A, Deluce A (1999) Local incremental planning for a car-like robot navigating among obstacle. Proceedings of IEEE Minnesota. April 1999

8. Almendia J, lobo J (2004) A hybrid system approach to feedback control of a nonholonomic car-like vehicle. IEEE Trans Autom Control 20(2):243-355

9. Yamaguchi H (2003) A distributed motion coordination strategy for multiple nonholonomic mobile robots in cooperative hunting operations. Robotics Auton Syst 43(4):257-282

10. Moshtagh N, Michael D, Jadbabaie A, Daniilidis K (Aug 2009) Vision-based, distributed control laws for motion coordination of nonholonomic robots. IEEE Trans Robot 25(4):851-860

11. Pinto G (2007) Towards a control architecture for cooperative nonholonomic mobile robots. Doctoral Thesis, 26 Nov 2007. http://tel. archivesouvertes.fr/docs/00/19/38/35/PDF/thesis.pdf

12. Pinto AG, Fraissea P, Zapata R (2006) A decentralized algorithm to adaptive trajectory planning for a group of nonholonomic mobile robots. IEEE/RSJ international conference on intelligent robots and systems, pp 404-417
13. Laumond JP, Jacobs PE (1994) A motion planner for nonholonomic mobile robots. IEEE Trans Robot Autom 10(5):577-593

14. Xi W, Baras JS (2007) MPC based motion control of car-like vehicle swarms. Proceeding of the 15th Mediterranean Conference on Control \& Automation, Athens, Greece, pp 1-6, July 27-29 2007

15. Pinto G, Fraisse P, Zapata (2007) Decentralized strategy for car-like robot formations. IEEE/RSJ international conference on intelligent robots and systems, pp 4176-4181

16. Baruh H (1999) Analitycal dynamics, Chap 7. McGraw -Hill, New York

17. Donald T (2003) Greenwood, Chap 4. Advansed dynamics. Cambridge University Press, Cambridge

18. Bowska EJ (2009) Stabilizability and motion tracking conditions for mechanical nonholonomic control systems. Math Probl Eng. doi: $10.1155 / 2007 / 31267$

19. Luc AD, Oriolo G (1995) Modeling and control of nonholonomic mechanical systems. Kinematics and dynamics of multi-body systems, vol 360. Springer, Wien, pp 277-342

20. Urcola P, Riazuelo L (2008) Cooperative navigation using environment compliant robot formations. IEEE/RSJ international conference on intelligent robots and systems. Acropolis Convention Center Nice, France, pp. 2789-2794, 22-26 Sept 2008

21. Simon D (1976) Optimal state estimation Kalman, H_infinity and nonlinear approaches. Cleveland State University, Wiley, Hoboken, NJ

22. Murphy K (1998) Kalman filter and smoother toolbox. http://www. ai.mit.edu/ murphyk/Software/

23. Särkkä S (2011) Bayesian estimation of time-varying systems: discrete-time systems. Version 1.2, 22 Jan 2011

24. Hartikainen J, Solin A, Särkkä A (2011) Optimal filtering with Kalman filters and smoothers, Version 1.3.16. Department of Biomedical Engineering and Computational Science, 16 Aug 2011

25. Wang Y, Wang S, Tan R, Jiang Y (2013) Motion control of a wheeled mobile robot using digital acceleration control method. Int J Innov Comput Inf Control (ICIC). 9(1) 